\title{
Diophantine Equations in Many Variables
}

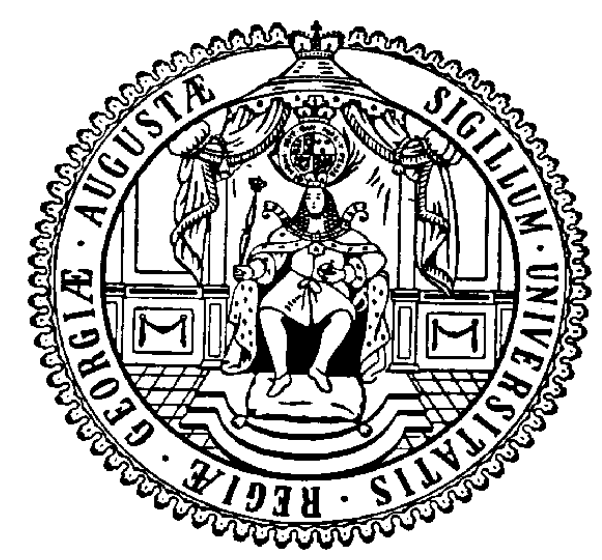

Dissertation

zur Erlangung des mathematisch-naturwissenschaftlichen Doktorgrades

"Doctor rerum naturalium"

der Georg-August-Universität Göttingen

im Promotionsprogramm der PhD School of Mathematical Science (SMS) der Georg-August University School of Science (GAUSS).

vorgelegt von

Jan Henrik Dumke

aus Hamburg

Göttingen, 2014 
Betreuungsausschuss

Prof. Dr. Jörg Brüdern,

Mathematisches Institut,

Georg-August-Universität Göttingen

Prof. Dr. Valentin Blomer,

Mathematisches Institut,

Georg-August-Universität Göttingen

Mitglieder der Prüfungskommission

Prof. Dr. Laurent Bartholdi,

Mathematisches Institut,

Georg-August-Universität Göttingen

Prof. Dr. Preda Mihăilescu,

Mathematisches Institut,

Georg-August-Universität Göttingen

Prof. Dr. Gerlind Plonka-Hoch,

Institut für Numerische und Angewandte Mathematik,

Georg-August-Universität Göttingen

Prof. Dr. Dominic Schuhmacher,

Institut für Mathematische Stochastik,

Georg-August-Universität Göttingen

Tag der mündlichen Prüfung: 8. Oktober 2014 

Contents

Abstract ii

Zusammenfassung (German abstract) iii

$\begin{array}{lr}\text { 1. Introduction } & 1\end{array}$

2. Schmidt's Minimisation Argument 5

3. Zeros of Quintic Forms 13

4. Svstems comprising a Cubic and a Quadratic Form 24

$\begin{array}{ll}\text { 5. Appendix } & 27\end{array}$

References 33 


\section{ABSTRACT}

Let $\mathbb{K}$ denote a $p$-adic field and $F_{1}, \ldots, F_{r} \in \mathbb{K}\left[x_{1}, \ldots, x_{n}\right]$ be forms with respective degrees $d_{1}, \ldots, d_{r}$. A contemporary version of a conjecture attributed to $\mathrm{E}$. Artin states that $F_{1}, \ldots, F_{r}$ have a common non-trivial zero whenever

$$
n>d_{1}^{2}+\cdots+d_{r}^{2}
$$

We prove this for a single quintic form (i.e. $r=1, d_{1}=5$ ), provided that the cardinality of the residue class field exceeds 9 . We also verify the conjecture for a system comprising a cubic and a quadratic form (i.e. $r=2, d_{1}=3, d_{2}=2$ ), whenever the residue class field is of characteristic at least 13 and has more than 37 elements. 


\section{ZUSAMMENFASSUNG}

Seien $\mathbb{K}$ ein $p$-adischer Körper und $F_{1}, \ldots, F_{r} \in \mathbb{K}\left[x_{1}, \ldots, x_{n}\right]$ Formen mit dazugehörigen Graden $d_{1}, \ldots, d_{r}$. Eine Vermutung, die auf E. Artin zurückgeführt wird, besagt in moderner Fassung, dass $F_{1}, \ldots, F_{r}$ eine nicht triviale gemeinsame Nullstelle besitzen falls

$$
n>d_{1}^{2}+\cdots+d_{r}^{2} .
$$

Wir beweisen dies im Falle einer einzelnen quintischen Form, vorausgesetzt die Kardinalität des Restklassenkörpers ist größer als 9. Zudem verifizieren wir die Vermutung für ein System bestehend aus einer kubischen und einer quadratischen Form, falls der Restklassenkörper mindestens Charakteristik 13 und mehr als 37 Elemente hat. 


\section{ACKNOWLEDGEMENTS}

I am grateful to my supervisor Professor Jörg Brüdern for his guidance, encouragement and support during my time as a PhD student at the University of Göttingen.

I would also like to express my gratitude to Professor Valentin Blomer for co-supervising this work.

This thesis has been partly written while the author was visiting the Mathematical Institute at the University of Oxford. Its hospitality and the provided very pleasant working environment are much appreciated. I have benefited from numerous conversations with Professor Roger Heath-Brown who made my stay possible and to whom the author is delighted to express his gratitude.

I also like to thank my colleagues at the Mathematical Institute in Göttingen for their help.

Finally, I am in debt to friends and family who have enriched my life in so many ways. 


\section{INTRODUCTION}

Let $\mathbb{K}$ be a finite extension of the field $\mathbb{Q}_{p}$ and $F_{1}, \ldots, F_{r} \in \mathbb{K}\left[x_{1}, \ldots, x_{n}\right]$ be forms with respective degrees $d_{1}, \ldots, d_{r}$. A modern version of an old conjecture attributed to E. Artin states that $F_{1}, \ldots, F_{r}$ have a common non-trivial zero whenever

$$
n>d_{1}^{2}+\cdots+d_{r}^{2}
$$

Unfortunately, this has only been confirmed for a single quadratic form (Hasse [13]), a single cubic form (Lewis [19]) and a system comprising two quadratic forms (Dem'yanov [9] and independently Birch, Lewis and Murphy [4]). In fact counterexamples are known for many degrees $d_{1}, \ldots, d_{r}$. The first counterexample was given by Terjanian [22]. He has found the explicit form

$$
\begin{aligned}
& G\left(x_{1}, x_{2}, x_{3}\right)+G\left(x_{4}, x_{5}, x_{6}\right)+G\left(x_{7}, x_{8}, x_{9}\right) \\
& +4 G\left(x_{10}, x_{11}, x_{12}\right)+4 G\left(x_{13}, x_{14}, x_{15}\right)+4 G\left(x_{16}, x_{17}, x_{18}\right)
\end{aligned}
$$

where

$$
G\left(x_{1}, x_{2}, x_{3}\right)=\sum_{i=1}^{3} x_{i}^{4}-\sum_{0 \leq i<j \leq 3} x_{i}^{2} x_{j}^{2}-x_{1} x_{2} x_{3}\left(x_{1}+x_{2}+x_{3}\right)
$$

that does not have a non-trivial zero over the 2-adic numbers.

Although false in general the conjecture has been partially verified by Ax and Kochen [1]. They showed that for every tuple $\left(d_{1}, \ldots, d_{r}\right)$ and degree $\eta$ of the field extension $\mathbb{K} / \mathbb{Q}_{p}$ there exists a positive integer $q_{0}\left(d_{1}, \ldots, d_{r}, \eta\right)$, such that Artin's Conjecture for forms of degrees $d_{1}, \ldots, d_{r}$ holds whenever the cardinality $q$ of the residue class field exceeds $q_{0}\left(d_{1}, \ldots, d_{r}, \eta\right)$. However, little is known about the actual values of $q_{0}\left(d_{1}, \ldots, d_{r}, \eta\right)$. For a single form of degree $d$ Brown [6] has given a huge, but explicit bound. If we write $a \uparrow b$ for $a^{b}$ it can be stated as

$$
q_{0}(d, 1) \leq 2 \uparrow(2 \uparrow(2 \uparrow(2 \uparrow(2 \uparrow(d \uparrow(11 \uparrow(4 d))))))) .
$$

This was intended as a neat rather than best possible expression, but optimising the argument is expected to lead to similar magnitudes.

If $d$ is neither composite nor a sum of composite numbers, better bounds are available. Since these hold independently of $\left[\mathbb{K}: \mathbb{Q}_{p}\right]$ we shall omit $\eta$ in our notion of $q_{0}$. Besides the classical result $q_{0}(2)=1$ and $q_{0}(3)=1$ this concerns in fact $d=5,7,11$ only. All other degrees are composite or a sum of composite numbers. Leep and Yeomans [18 have shown $q_{0}(5) \leq 43$ and later this has been improved by Heath-Brown [14]. He proved that a quintic form over $\mathbb{Q}_{p}$ possesses a non-trivial zero, provided $p \geq 17$. For septic and undecic forms bounds $q_{0}(7) \leq 883$ and 
$q_{0}(11) \leq 8053$ are due to Wooley [24].

These results use a $p$-adic minimisation procedure which has been developed by Lewis [19], Birch and Lewis [2] and Laxton and Lewis [16] and ultimately rely on lifting a non-singular zero via Hensel's Lemma.

In this thesis we shall establish the result $q_{0}(5) \leq 9$. In order to ease notation, we will from now on call a finite extension of the field $\mathbb{Q}_{p}$ a $p$-adic field.

Theorem 1. Let $F\left(x_{1}, \ldots, x_{n}\right)$ be a quintic form with at least $n \geq 26$ variables over a $p$-adic field $\mathbb{K}$ with residue class field of cardinality $q>9$. Then there exists a non-zero vector $\mathbf{x} \in \mathbb{K}^{n}$ with $F(\mathbf{x})=0$.

As we are interested in a zero, we may assume that $F$ is defined over the ring of integers $\mathcal{O}_{\mathbb{K}}$. The proof is based on a $p$-adic minimisation procedure which has been developed by Schmidt [20] and generalised by Zahid [25]. By applying their technique we may assume that the reduction of $F$ over the residue class field, denoted by $\theta(F)$, is a nondegenerate form with at least $6+s$ variables, where $s$ is the maximal affine dimension of a vector space on which $\theta(F)$ vanishes 1 If $\theta(F)$ possesses a non-singular zero, it can be lifted by Hensel's Lemma to a non-trivial zero of $F$. We recall that a non-singular zero is one which is not a simultaneous zero of the partial derivatives.

We shall use certain properties of quintic forms to choose a suitable subspace and show that it contains a non-singular zero. For $q=$ $11,13,16,25,27,32$ this is accomplished with the help of computer calculations 2 The author was able to carry those out on his personal notebook. This, together with the previously mentioned results of Leep and Yeomans and Heath-Brown, yields Theorem 1.

There is numerical evidence to suggest that the imposed constraint on $q$ can be further reduced. Given the current state of technology, it certainly seems doubtful to expect an answer for all $q$ at this stage.

On the other hand there are a number of results in the same vein concerning systems of forms. E. Schuur 21, improving on work of Birch and Lewis [3], has shown that three quadratic forms have a common non-trivial zero if the cardinality of the residue class field exceeds 9. Heath-Brown [15] has established Artin's Conjecture for a system of $r$ quadratic forms, provided $q>(2 r)^{r}$. Recently Zahid [26] has been

\footnotetext{
${ }^{1}$ Precise definitions of " $s$ " and "non-degenerate" can be found at the beginning of Chapter 3 ,

${ }^{2}$ For $q=25,27$ and 32 a false attempt of proof was made in Theorem 7 of the author's master's thesis.
} 
the first addressing forms of differing degrees by proving $q_{0}(3,2) \leq 293$.

We shall establish the following improvement, provided the characteristic of the residue class field is at least 13 .

Theorem 2. Let $C\left(x_{1}, \ldots, x_{n}\right)$ be a cubic and $Q\left(x_{1}, \ldots, x_{n}\right)$ a quadratic form with at least $n \geq 14$ variables over a $p$-adic field $\mathbb{K}$ with residue class field of cardinality $q>37$ and characteristic at least 13 . Then there exists a non-zero vector $\mathbf{x} \in \mathbb{K}^{n}$ with $C(\mathbf{x})=Q(\mathbf{x})=0$.

In order to prove Theorem 2 we follow the path laid by Zahid. By exploiting Schmidt's minimisation procedure Zahid extracts information on the reduction of the system over the residue class field. He then shows that $\theta(C)$ and $\theta(Q)$ have a common non-singular zero, provided a certain absolutely irreducible quartic form $H \in \mathbb{F}_{q}\left[x_{2}, \ldots, x_{n}\right]$ possesses a non-singular and $q>5$. Recall that a polynomial over a field is absolutely irreducible if it is irreducible over the algebraic closure of the field. Also note that a common non-singular zero of forms $f_{1}, \ldots, f_{r}$ over $\mathbb{F}_{q}$ is a zero $\mathbf{x}$ such that $\nabla f_{1}(\mathbf{x}), \ldots, \nabla f_{r}(\mathbf{x})$ are linearly independent. By a slicing argument there exists a vector $\xi \in \mathbb{F}_{q}^{3 n-5}$ such that

$\left.H\right|_{\xi}(X, Y):=H\left(\xi_{1}+X, \xi_{2}+\xi_{n} X+\xi_{2 n-4} Y, \ldots, \xi_{n-1}+\xi_{2 n-3} X+\xi_{3 n-5} Y\right)$

remains absolutely irreducible, provided $q>296$. Using the Lang-Weil Bound he concludes that $\left.H\right|_{\xi}$, and hence $H$, has a non-singular zero. The improvement in Theorem 2 is due to a more effective slicing process. Using a quantitative Bertini theorem by Lecerf [17] we obtain $\xi$ as above yet require a much weaker condition on the cardinality of the residue class field, videlicet $q>37$.

An alternative approach towards Artin's Conjecture is to ask for the minimal number of variables needed to ensure the existence of a nontrivial zero. Brauer [5] has shown that there is a finite non-negative integer $v\left(d_{1}, \ldots, d_{r}\right)$ such that $F_{1}, \ldots, F_{r}$ possess a common non-trivial zero whenever

$$
n \geq v\left(d_{1}, \ldots, d_{r}\right)
$$

His proof reduces the problem to diagonal forms, which have been studied extensively (see in particular [8]). Refined subsequent results use quasi-diagonalisation techniques. The best general bound is due to Wooley [23]. For a system comprising $r$ forms of degree $d$ over $\mathbb{Q}_{p}$ he 
showed that $n>\left(r d^{2}\right)^{2^{d-1}}$ suffices 3

For a number of degrees better bounds are available. Firstly, we can extract better estimates from Wooley's proof for specific $d$. Secondly, Heath-Brown [14] considerably improved the bound for a single quartic form by establishing $v_{\mathbb{Q}_{p}}(4) \leq 4222$. Here we write $v_{\mathbb{Q}_{p}}$ to indicate the restriction to forms over $\mathbb{Q}_{p}$. His proof has been adapted by Zahid [25] to show $v_{\mathbb{Q}_{p}}(5) \leq 4562912$.

Heath-Brown's method provides better results if the involved degrees are not multiples of $p$. The author has developed a variant yielding improved bounds if $p$ does divide the degree (see [11]). As an example, he proves that $v_{\mathbb{Q}_{p}}(3,3) \leq 132$ and $v_{\mathbb{Q}_{p}}(4) \leq 3192$. To prove these bounds the author has by successively choosing and recycling vectors constructed a subspace on which the forms involved are of a special shape. It transpires that this can be done if systems comprising a certain number of quadratic and linear forms have a non-trivial zero. Finally, a version of Hensel's Lemma can be applied.

This method would enormously benefit from better results on systems of quadratics. In contrast, it is not hard to see that $v(2,2,2,2) \leq v(4)$.

This thesis is organised as follows. Chapter 2 gives an account of Schmidt's minimisation procedure on which all our results are based. In Chapter 3 a proof of Theorem 1 on a single quintic is presented. The case of a cubic and a quadratic form is discussed in Chapter 4. The final Chapter 5 provides some of the source code as used in the proof of Theorem 1. Extracts from this thesis have been made available online at [10] and [11]

\footnotetext{
${ }^{3}$ Wooley also gives bounds for the more general situation of forms over $p$-adic fields.

${ }^{4}$ In [11, this only concerns the introduction.
} 


\section{Schmidt's Minimisation Argument}

In this chapter we shall introduce a powerful minimisation argument by Schmidt [20]. We will, however, follow a more recent and generalised account by Zahid [26]. His version is the first that extends to systems comprising forms of differing degrees, which allows us to discuss common zeros of a cubic and a quadratic form in Chapter 4. The reader familiar with this argument or chiefly interested in the new ideas involved in the proof of Theorem 1 or Theorem 2 is referred to Chapter 3 or Chapter 4, respectively.

2.1. Statement of the main theorem. Let $\mathbb{K}$ denote a $p$-adic field with residue class field $\mathbb{F}_{q}$ and ring of integers $\mathcal{O}_{\mathbb{K}}$. We shall write $\pi$ for a uniformiser of $\mathcal{O}_{\mathbb{K}}$. Recall that every non-zero $a \in \mathbb{K}$ can be uniquely written as $a=u \pi^{l}$, where $u \in \mathcal{O}_{\mathbb{K}}$ is a unit and $l \geq 0$, and that its normalised valuation is given by $\nu(a)=l$. Also, note that every non-zero $a \in \mathbb{K}$ has norm $|a|=p^{-\nu(a)}$.

Let $\mathbf{F}=\left(F_{1}, \ldots, F_{r}\right)$ be a system of forms over $\mathbb{K}$ in $n$ variables comprising $r$ forms of degree $d_{1} \geq \cdots \geq d_{r}$. As we are interested in a zero, we may assume from now on that $F_{1}, \ldots, F_{r}$ are defined over $\mathcal{O}_{\mathbb{K}}$.

Before we can state the main theorem we need to introduce a few definitions. Let $A$ be an $n \times n$-matrix over $\mathcal{O}_{\mathbb{K}}$ and $B$ be an upper-triangular $r \times r$-matrix with entries

$$
(B)_{i j}=\pi^{-c_{i}} G_{i j}
$$

where $c_{i}$ is a non-negative integer, $G_{i i}=1$ and otherwise $G_{i j} \in \mathcal{O}_{\mathbb{K}}\left[x_{1}, \ldots, x_{n}\right]$ any form such that

$$
\operatorname{deg}\left(G_{i j}\right)=\operatorname{deg}\left(F_{i}\right)-\operatorname{deg}\left(F_{j}\right) .
$$

We say that $\mathbf{F}$ and another system $\hat{\mathbf{F}}$ of $r$ forms over $\mathcal{O}_{\mathbb{K}}$ with $n$ variables are equivalent if there exist $A, B$ as described above, but $c_{i}=0$ for all $1 \leq i \leq r$, such that

$$
\mathbf{F}(\mathbf{x})=B \hat{\mathbf{F}}(A \mathbf{x}) \quad \text { and } \quad \nu(\operatorname{det}(A))=0 .
$$

We are now relaxing the last condition and allowing any non-negative integer values for $c_{i}$ for all $1 \leq i \leq r$. For $\mathbf{F}, \hat{\mathbf{F}}$ and a vector $\Omega=$ $\left(\omega_{1}, \ldots, \omega_{r}\right)$ with positive rational

$$
\mathbf{F} \succsim_{\Omega} \hat{\mathbf{F}}
$$

\footnotetext{
${ }^{5} \mathrm{In}[26$, it is not mentioned that $\Omega$ is required to be a vector of rational numbers. This, however, is crucial for the proof of Lemma 1
} 
if there are $A$ and $B$ as in (2) such that

$$
\mathbf{F}(\mathbf{x})=B \hat{\mathbf{F}}(A \mathbf{x}) \quad \text { and } \quad \sum_{i=1}^{r} c_{i} \omega_{i}-\nu(\operatorname{det}(A))>0 .
$$

If this holds, $\mathbf{F}$ possesses a non-trivial zero if and only if $\hat{\mathbf{F}}$ does. With this key definition at hand we declare a system $\mathbf{F}$ to be $\Omega$-bottomless if there exists an infinite sequence of systems $\left(\mathbf{F}_{i}\right)_{i}$ such that

$$
\begin{array}{lllllllll}
\mathbf{F} & \succ & \mathbf{F}_{1} & \succ & \mathbf{F}_{2} & \succ & \mathbf{F}_{3} & \ldots
\end{array} .
$$

A system that is not $\Omega$-bottomless is said to be $\Omega$-bottomed. An $\Omega$ bottomed system $\mathbf{F}$ is called $\Omega$-reduced if there does not exist a system $\hat{\mathbf{F}}$ such that

$$
\mathbf{F} \succ_{\Omega} \hat{\mathbf{F}} .
$$

For any finite set of positive integers $S=\left\{d_{1}, \ldots, d_{s}\right\}$ we set

$$
v(S):=v\left(d_{1}, \ldots, d_{s}\right) .
$$

As defined in the introduction, this is the least integer such that any system comprising forms of degree $d_{1}, \ldots, d_{s}$ has a non-trivial zero. We are now in the position to state the main result of the minimisation procedure as proved by Zahid.

Theorem 3 (Theorem 2.1, [26]). Let $S \subset\left\{d_{1}, \ldots, d_{r}\right\}$ denote any subset of cardinality $r-1$ with indexing set I such that $v(S)$ is maximal. Also let $d \in\left\{d_{1}, \ldots, d_{r}\right\}-S$. Then, provided that

$$
n \geq v(S)+d^{2}
$$

there exists some $\Omega=\left(\omega_{1}, \ldots, \omega_{r}\right) \in \mathbb{Q}_{>0}^{r}$ such that $\omega_{i}>d_{i}$ for each $1 \leq i \leq r$ and such that every $\Omega$-bottomless system $\mathbf{F}$ defined over $\mathcal{O}_{\mathbb{K}}$ has a p-adic zero.

2.2. Proof of Theorem 3. Zahid's proof relies on the fact that every $\Omega$-bottomless system is equivalent to a so-called $\Omega$-special system. It is then shown that any $\Omega$-special system possesses a non-trivial zero. We will follow his account [26] closely.

In order to introduce the notion of an $\Omega$-bottomless system, we associate with a form $F_{i}$ a unique symmetric multilinear form $M_{F_{i}}\left(\mathbf{x}_{1}, \ldots, \mathbf{x}_{d_{i}}\right)$ such that

$$
F_{i}(\mathbf{x})=M_{F_{i}}(\mathbf{x}, \ldots, \mathbf{x})
$$


As $\mathbb{K}$ is of characteristic zero, there always exists such a unique form. $\mathbf{F}$ is called $\Omega$-special if there are non-negative integers $a_{1}, \ldots, a_{n}$ and $b_{1}, \ldots, b_{r}$ satisfying the relation

$$
a_{1}+\cdots+a_{n}<\omega_{1} b_{1}+\cdots+\omega_{r} b_{r}
$$

such that for each tuple of standard basis vectors $\mathbf{e}_{j_{1}}, \ldots, \mathbf{e}_{j_{d_{i}}}$ one has

$$
M_{F_{i}}\left(\mathbf{e}_{j_{1}}, \ldots, \mathbf{e}_{j_{d_{i}}}\right)=0
$$

whenever $1 \leq i \leq r$ and

$$
a_{j_{1}}+\cdots+a_{j_{d_{i}}}<b_{i}
$$

Theorem 4 (Theorem 3.1, [26]). Every $\Omega$-bottomless system is equivalent to an $\Omega$-special system.

Instead of proving Theorem 4 now, we shall postpone this for a short while and deduce Theorem 3 first.

2.2.1. Deriving Theorem 3 from Theorem 4. Suppose that $\mathbf{F}$ is an $\Omega$ bottomless system. By Theorem 4 we may assume that $\mathbf{F}$ is $\Omega$-special. Let $a_{1}, \ldots, a_{n}$ and $b_{1}, \ldots, b_{r}$ be as defined in (5)-(7). For convenience we shall assume that

$$
a_{1} \leq \cdots \leq a_{n} \quad \text { and } \quad \frac{b_{1}}{d_{1}} \leq \cdots \leq \frac{b_{r}}{d_{r}} .
$$

Note that one might have to rearrange the ordering of $d_{1}, \ldots, d_{r}$. Let $I \subseteq\{1, \ldots, r\}$ be a set indexing $\left\{F_{i}\right\}_{i \in I}$ and $S \subset\left\{d_{1}, \ldots, d_{r}\right\}$ the corresponding set of degrees. If there exists $I$ such that

$$
d_{i} a_{v(S)}<b_{i}
$$

for all $i \notin I$, then relation (7) holds and, consequently, (6) yields

$$
M_{F_{i}}\left(\mathbf{e}_{j_{1}}, \ldots, \mathbf{e}_{j_{d_{i}}}\right)=0
$$

for every $i \notin I$ and $1 \leq j_{1}, \ldots, j_{d_{i}} \leq v(S)$. Thus, $\left\{F_{i}\right\}_{i \notin I}$ vanishes on a vector space of dimension $v(S)$. By definition $\left\{F_{i}\right\}_{i \in I}$ has a non-trivial zero in this vector space. We conclude that $\mathbf{F}$ possesses a non-trivial zero.

Otherwise, we show that there exists a suitable $\Omega$ such that $\mathbf{F}$ is not $\Omega$-special. If (9) fails for all $I$, then there must for every $I$ be a certain $i \in I$ such that

$$
d_{i} a_{v(S)} \geq b_{i} .
$$

If we write $S_{0}=\emptyset$ and $S_{i}=\left\{d_{1}, \ldots, d_{i}\right\}$ for all $1 \leq i \leq r-1$, then (10) yields

$$
d_{i} a_{v\left(S_{i-1}\right)} \geq b_{i} \quad \text { for all } 1 \leq i \leq r .
$$


If both a system comprising forms $F_{1}, \ldots, F_{i-1}$ in $v\left(S_{i-1}\right)-1$ variables and a single form $F_{i}$ in $v\left(d_{i}\right)-1$ variables, distinct from those of $F_{1}, \ldots, F_{i-1}$, do not possess a non-trivial zero, then neither does the system $F_{1}, \ldots, F_{i}$. In other words

$$
v\left(S_{i}\right)-v\left(S_{i-1}\right) \geq v\left(d_{i}\right)-1 .
$$

Since $v\left(d_{i}\right) \geq d_{i}^{2}+1$ (see 6 ) we have

$$
v\left(S_{i}\right)-v\left(S_{i-1}\right) \geq d_{i}^{2} .
$$

Also note that assumption (4) implies

$$
n>d_{1}^{2}+\cdots+d_{r}^{2} \text {. }
$$

By (12), the assumed ordering (8) and (13), we have

$$
\begin{aligned}
a_{1}+\cdots+a_{n} & \geq a_{1}+\cdots+a_{d_{1}^{2}}+\cdots+a_{d_{r}^{2}}+a_{n} \\
& \geq a_{v\left(S_{0}\right)} d_{1}^{2}+\cdots+a_{v\left(S_{r-1}\right)} d_{r}^{2}+a_{n} .
\end{aligned}
$$

Since $a_{n} \geq\left(a_{1}+\cdots+a_{r}\right) / r$ this is greater than or equal to

$$
\begin{aligned}
& \left(d_{1}^{2}+\frac{1}{r}\right) a_{v\left(S_{0}\right)}+\left(d_{2}^{2}+\frac{1}{r}\right) a_{v\left(S_{1}\right)}+\cdots+\left(d_{r}^{2}+\frac{1}{r}\right) a_{v\left(S_{r-1}\right)} \\
\geq & \left(d_{1}+\frac{1}{r \max _{1 \leq i \leq r} d_{i}}\right) b_{1}+\cdots+\left(d_{r}+\frac{1}{r \max _{1 \leq i \leq r} d_{i}}\right) b_{r}
\end{aligned}
$$

where the last inequality follows from equation (11). By setting

$$
\omega_{i}:=d_{i}+1 / r \max _{1 \leq i \leq r}\left(d_{i}\right),
$$

we have a suitable $\Omega$ such that $\mathbf{F}$ is not $\Omega$-special.

Thus we conclude that there is a suitable $\Omega$ such that $\mathbf{F}$ has a nontrivial $p$-adic zero, provided $\mathbf{F}$ is $\Omega$-bottomless.

2.2.2. Preparations for the proof of Theorem 4. Suppose that $\mathbf{F}$ and $\hat{\mathbf{F}}$ are systems comprising $r$ forms over $\mathcal{O}_{\mathbb{K}}$. For a vector $\Omega=\left(\omega_{1}, \ldots, \omega_{r}\right)$ with positive rational components and an integer $k \geq 1$ we write

$$
\mathbf{F} \quad \succ_{\Omega}^{k} \hat{\mathbf{F}}
$$

if there are $A$ and $B$ as described in (2) such that

$$
\mathbf{F}(\mathbf{x})=B \hat{\mathbf{F}}(A \mathbf{x}) \quad \text { and } \quad \sum_{i=1}^{r} c_{i} \omega_{i}-\nu(\operatorname{det}(A)) \geq k .
$$

\footnotetext{
${ }^{6}$ Note that for every positive integer $d$ there exists a form of degree $d$ with $d^{2}$ variables which does not have a non-trivial zero. This follows, for instance, from the existence of a central simple division algebra of rank $d^{2}$.
} 
This strengthens the definition of " $\succ$ " as given in (3). We say that a system of forms $\mathbf{F}$ is $\Omega$-high if for every integer $k \geq 1$ there exists a system $\hat{\mathbf{F}}$ such that

$$
\mathbf{F} \quad \overbrace{\Omega}^{k} \hat{\mathbf{F}} .
$$

Lemma 1 (Lemma 3.2, [26]). If $\mathbf{F}$ is an $\Omega$-bottomless system, then it is $\Omega$-high.

Proof. Suppose that $\mathbf{F}_{1}$ is an $\Omega$-bottomless system. Consequently, there exists an infinite sequence $\left(\mathbf{F}_{i}\right)_{i \geq 2}$ of systems such that

$$
\begin{array}{llllllllll}
\mathbf{F}_{1} & \succ & \mathbf{F}_{2} & \succ & \mathbf{F}_{3} & \succ & \mathbf{F}_{4} & \succ & \ldots
\end{array} .
$$

By definition there exist for each $m \geq 1$ matrices $A_{m}$ and $B_{m}$ as in (2) such that

$$
\mathbf{F}_{m}(\mathbf{x})=B_{m} \mathbf{F}_{m+1}\left(A_{m} \mathbf{x}\right) \quad \text { and } \quad \sum_{i=1}^{r} c_{i, m} \omega_{i}-\nu\left(\operatorname{det}\left(A_{m}\right)\right)>0 .
$$

Let $k$ be an arbitrary positive integer. By (15) and since $\Omega \in \mathbb{Q}_{>0}^{r}$ there exists a non-negative integer $Q$ such that

$$
\sum_{i=1}^{r} c_{i, m} \omega_{i}-\nu\left(\operatorname{det}\left(A_{m}\right)\right) \geq \frac{1}{Q}
$$

for all $1 \leq m \leq k Q$. By setting

$$
A:=\prod_{m=1}^{k Q} A_{m}, \quad B:=\prod_{m=1}^{k Q} B_{m} \quad \text { and } \quad c_{i}:=\sum_{m=1}^{k Q} c_{i, m}
$$

we have

$$
\mathbf{F}_{1}(\mathbf{x})=B \mathbf{F}_{k Q}(A \mathbf{x})
$$

and

$$
\sum_{i=1}^{r} c_{i} \omega_{i}-(\nu(\operatorname{det}(A))+k)=\sum_{m=1}^{k Q}\left(\sum_{i=1}^{r} c_{i, m} \omega_{i}-\nu\left(\operatorname{det}\left(A_{m}\right)\right)\right)-k \geq 0 .
$$

Thus we conclude that

$$
\mathbf{F}_{1} \quad \underset{\Omega}{\ell} \mathbf{F}_{k Q}
$$

We shall need two additional and slightly technical lemmas to finish the proof of Theorem 4, but skip their proofs. The reader interested in these is referred to lemmas 8 and 10 as found in [20]. 
Lemma 2 (Lemma 3.3, [26]). Let $C_{1}, \ldots, C_{l}$ and $D_{1}, \ldots, D_{m}$ be linear forms with integer coefficients in $\mathcal{O}_{\mathbb{K}}$ in the vector $\mathbf{x}=\left(x_{1}, \ldots, x_{n}\right)$. Further, let $\mathbf{x}_{1}, \mathbf{x}_{2}, \ldots$ be a sequence of vectors such that for all $1 \leq$ $i \leq l$ and $k \geq 1$

$$
C_{i}\left(\mathbf{x}_{k}\right) \geq 0
$$

Then there exists a subsequence, $\mathbf{y}_{1}, \mathbf{y}_{2}, \ldots$ say, a constant $D$ and an integer vector a with

$$
C_{i}(\mathbf{a}) \geq 0
$$

for all $1 \leq i \leq l$ such that

$$
\lim _{k \mapsto \infty} D_{j}\left(\mathbf{y}_{k}\right)=+\infty \quad \text { for } j \text { with } D_{j}(\mathbf{a})>0
$$

and

$$
D_{j}\left(\mathbf{y}_{k}\right) \leq D \quad \text { for } j \text { with } D_{j}(\mathbf{a}) \leq 0 .
$$

For the next lemma, we recall the notion of a lattice. Let $\mathbf{a}_{1}, \ldots, \mathbf{a}_{s} \in$ $\mathbb{K}^{n}$ be linearly independent vectors, then

$$
\Lambda=\left\{\lambda_{1} \mathbf{a}_{1}+\cdots+\lambda_{s} \mathbf{a}_{s} \mid\left(\lambda_{1}, \ldots, \lambda_{s}\right) \in \mathcal{O}_{\mathbb{K}}^{s}\right\}
$$

is called a lattice with basis $\mathbf{a}_{1}, \ldots, \mathbf{a}_{s}$. We say that a lattice $\hat{\Lambda}$ is a sub-lattice of $\Lambda$ if $\hat{\Lambda} \subseteq \Lambda$.

Lemma 3 (Lemma 3.4, 26]). Suppose that $M$ is a sub-lattice of $\Lambda$. Then there exists a basis $\mathbf{u}_{1}, \ldots, \mathbf{u}_{s}$ of $\Lambda$ and a basis of $\mathbf{m}_{1}, \ldots, \mathbf{m}_{s}$ of $M$ such that

$$
\mathbf{m}_{1}=\pi^{u_{1}} \mathbf{u}_{1}, \ldots, \mathbf{m}_{s}=\pi^{u_{s}} \mathbf{u}_{s},
$$

for some non-negative integers $u_{1}, \ldots, u_{s}$.

2.2.3. Proof of Theorem 4. Suppose that $\mathbf{F}$ is an $\Omega$-bottomless system over $\mathcal{O}_{\mathbb{K}}$ comprising $r$ forms in $n$ variables. By Lemma 1 we may assume that $\mathbf{F}$ is $\Omega$-high. By definition this means that for every $k \geq 1$ there is an $n \times n$-matrix $A_{k}$ over $\mathcal{O}_{\mathbb{K}}$ and an upper-triangular $r \times r$-matrix $B_{k}$ with entries

$$
\left(B_{k}\right)_{i j}=\pi^{-c_{i, k}} G_{i j, k}
$$

where $c_{i, k} \geq 0, G_{i i, k}=1$ and otherwise $G_{i j, k} \in \mathcal{O}_{\mathbb{K}}\left[x_{1}, \ldots, x_{n}\right]$ any form with

$$
\operatorname{deg}\left(G_{i j, k}\right)=\operatorname{deg}\left(F_{i}\right)-\operatorname{deg}\left(F_{j}\right)
$$


and a system $\mathbf{F}_{k}$ such that

$$
\mathbf{F}(\mathbf{x})=B_{k} \mathbf{F}_{k}\left(A_{k} \mathbf{x}\right) \quad \text { and } \quad \sum_{i=1}^{r} c_{i, k} \omega_{i}-\nu\left(\operatorname{det}\left(A_{k}\right)\right) \geq k .
$$

By defining a sequence of forms and taking the limit on a subsequence, we will construct an $\Omega$-special system equivalent to $\mathbf{F}$. For $1 \leq i \leq r$ set

$$
R_{i, k}(\mathbf{x}):=\sum_{j=i}^{r} G_{i j, k}(\mathbf{x}) F_{j}(\mathbf{x})
$$

Clearly, we have

$$
\pi^{-c_{i, k}} R_{i, k}(\mathbf{x}) \in \mathcal{O}_{\mathbb{K}}
$$

for any $\mathbf{x} \in A_{k} \mathcal{O}_{\mathbb{K}}^{n}$ and all $1 \leq i \leq r$. Note that $\mathcal{O}_{\mathbb{K}}^{n}$ is a lattice with sub-lattice $A_{k} \mathcal{O}_{\mathbb{K}}^{n}$. Thus, we can apply Lemma 3 and obtain a basis $\mathbf{m}_{1}, \ldots, \mathbf{m}_{n}$ of $A_{k} \mathcal{O}_{\mathbb{K}}^{n}$ and basis $\mathbf{u}_{1}, \ldots, \mathbf{u}_{n}$ of $\mathcal{O}_{\mathbb{K}}^{n}$ such that

$$
\mathbf{m}_{1}=\pi^{u_{1}} \mathbf{u}_{1}, \ldots, \mathbf{m}_{n}=\pi^{u_{n}} \mathbf{u}_{n}
$$

for some non-negative integers $u_{1}, \ldots, u_{n}$. As $\mathbb{K}$ is of characteristic zero, there exists for every $1 \leq i \leq r$ a unique multilinear form $M_{R_{i, k}}$ associated with $R_{i, k}$. Unfortunately, it does not necessarily follow from (17) that $M_{R_{i, k}}\left(\mathbf{x}_{1}, \ldots, \mathbf{x}_{d_{i}}\right) \in \mathcal{O}_{\mathbb{K}}$ for $\mathbf{x}_{1}, \ldots, \mathbf{x}_{d_{i}} \in \mathcal{O}_{\mathbb{K}}^{n}$ and $1 \leq i \leq r$. As $R_{i, k}$ is defined over $\mathcal{O}_{\mathbb{K}}$, there exists, however, $\gamma \in \mathbb{N}_{0}$, depending on $d_{1}, \ldots, d_{r}$, such that

$$
\pi^{\gamma} M_{R_{i, k}}\left(\mathbf{x}_{1}, \ldots, \mathbf{x}_{d_{i}}\right) \in \mathcal{O}_{\mathbb{K}}
$$

for all $\mathbf{x}_{1}, \ldots, \mathbf{x}_{d_{i}} \in \mathcal{O}_{\mathbb{K}}^{n}$ and $1 \leq i \leq r$. Using the basis vectors $\mathbf{m}_{1}, \ldots, \mathbf{m}_{n}$ from (18) we have

$$
\pi^{-c_{i, k}} M_{R_{i, k}}\left(\pi^{u_{j_{1}}} \mathbf{u}_{j_{1}}, \ldots, \pi^{u_{j_{d_{i}}}} \mathbf{u}_{j_{d_{i}}}\right) \in \pi^{-\gamma} \mathcal{O}_{\mathbb{K}}
$$

and hence

$$
\left|M_{R_{i, k}}\left(\mathbf{u}_{j_{1}}, \ldots, \mathbf{u}_{j_{d_{i}}}\right)\right| \leq p^{\gamma-\left(c_{i, k}-u_{j_{1}}-\ldots-u_{j_{d_{i}}}\right)}
$$

for every tuple $j_{1}, \ldots, j_{d_{i}}$ such that $1 \leq j_{1}, \ldots, j_{d_{i}} \leq n$. We stress that $\mathbf{u}_{i}, u_{i}$ are dependent on $k$ and that (19) holds for all $k$. Recall that $\mathbf{u}_{1}, \ldots, \mathbf{u}_{n}$ form a basis of the lattice $\mathcal{O}_{\mathbb{K}}^{n}$. Since $\mathcal{O}_{\mathbb{K}}^{n}$ is compact, there exists a convergent subsequence of $\left(\mathbf{u}_{1}, \ldots, \mathbf{u}_{n}\right)_{k}$ with limit $\left(\mathbf{a}_{1}, \ldots, \mathbf{a}_{n}\right)$. Note that $\mathbf{a}_{1}, \ldots, \mathbf{a}_{n}$ form again a basis of $\mathcal{O}_{\mathbb{K}}^{n}$. Moreover, $R_{1, k}, \ldots, R_{r, k}$ converge, on a subsequence again, to forms $R_{1}, \ldots, R_{r}$, say. There is a linear map $\sigma$ over $\mathcal{O}_{\mathbb{K}}$, such that

$$
\sigma \mathbf{e}_{i}=\mathbf{a}_{i}
$$


for all $1 \leq i \leq n$. In order to apply Lemma 2 we set for each $k$ in our subsequence

$$
\left(C_{1}, \ldots, C_{n+r}\right)_{k}:=\left(u_{1}, \ldots, u_{n}, c_{1, k}, \ldots, c_{r, k}\right) .
$$

Moreover we put, also for each $k$,

$$
D(k):=\sum_{i=1}^{r} c_{i, k} \omega_{i}-\sum_{i=1}^{n} u_{i}
$$

and write

$$
D_{i}(k)=c_{i, k}-\left(u_{j_{1}}+\cdots+u_{j_{d_{i}}}\right)
$$

where $1 \leq j_{1}, \ldots, j_{d_{i}} \leq n$ and $1 \leq i \leq r$. As

$$
\nu\left(\operatorname{det}\left(A_{k}\right)\right)=\sum_{i=1}^{n} u_{i}
$$

we have by (16)

$$
\sum_{i=1}^{k} c_{i, k} \omega_{i}-\sum_{i=1}^{n} u_{i} \geq k
$$

and therefore

$$
\lim _{k \rightarrow+\infty} D(k)=+\infty .
$$

It then follows from Lemma 2 that there is

$$
\mathbf{a}=\left(a_{1}, \ldots, a_{n}, b_{1}, \ldots, b_{r}\right)
$$

with $a_{i}$ for $1 \leq i \leq n$ and $b_{i}$ for $1 \leq i \leq r$ non-negative integers such that

$$
a_{1}+\cdots+a_{n}<\omega_{1} b_{1}+\cdots+\omega_{r} b_{r} .
$$

Moreover Lemma 2 states there is a subsequence such that $D_{i}(k)$ tends to $+\infty$ for all indices $i$ and $j_{1}, \ldots, j_{d_{i}}$, which satisfy

$$
a_{j_{1}}+\cdots+a_{j_{d_{i}}}<b_{i} \text {. }
$$

By letting $k$ in equation (19) tend to $+\infty$, we observe that for the same range of indices holds

$$
M_{R_{i}}\left(\mathbf{a}_{j_{1}}, \ldots, \mathbf{a}_{j_{d_{i}}}\right)=0
$$

Thus, we obtain with $\hat{\mathbf{F}}(\mathbf{x}):=\mathbf{F}(\sigma \mathbf{x})$ an $\Omega$-special system that is equivalent to $\mathbf{F}$. This completes the proof of Theorem 4 and ends our exposition of Zahid's account. 


\section{Zeros of Quintic Forms}

In this chapter we shall give a proof of Theorem 11. Firstly, we discuss some preliminary manoeuvres involving the minimisation procedure from Chapter 2, the Chevalley-Warning Theorem and a version of Hensel's Lemma.

3.1. Preliminary manoeuvres. We say that two forms $f$ and $g \in$ $\mathbb{F}_{q}\left[x_{1}, \ldots, x_{n}\right]$ are equivalent if there exist a non-zero $a \in \mathbb{F}_{q}$ and $A \in$ $\mathrm{GL}_{n}\left(\mathbb{F}_{q}\right)$ such that

$$
g(\mathbf{x})=a f(A \mathbf{x})
$$

If $f$ and $g$ are equivalent, then $f$ has a non-singular zero if and only if $g$ possesses one. A form $f$ over $\mathbb{F}_{q}$ is said to be non-degenerate if the number of variables explicit in $f$ is minimal among all forms equivalent to $f$.

Recall that $\mathbb{K}$ denotes a $p$-adic field with residue class field $\mathbb{F}_{q}$ and ring of integers $\mathcal{O}_{\mathbb{K}}$. As previously mentioned, we write $\pi$ for a uniformiser of $\mathcal{O}_{\mathbb{K}}$.

Let $F$ be a quintic form with at least 26 variables over $\mathbb{K}$. As we are interested in a zero, we may assume from now on that $F$ is defined over $\mathcal{O}_{\mathbb{K}}$.

We use Schmidt's minimisation procedure to derive some geometric information on the reduction of $F$ over the residue class field. Recall that the reduction of $F$ is denoted by $\theta(F)$. The next lemma extends Proposition 4.3 of [18] in the case of quintic forms. Note that there always exists a non-degenerate form equivalent to $\theta(F)$. In order to ease notation we assume without loss of generality that $\theta(F)$ is nondegenerate.

Lemma 4. Let $F$ be a quintic form in at least 26 variables over $\mathcal{O}_{\mathbb{K}}$ that does not have a non-trivial zero. Suppose that $\theta(F)$ is a nondegenerate form with $m$ variables $x_{1}, \ldots, x_{m}$ explicit in $\theta(F)$. Let $s \geq 0$ be an integer such that the form $\theta(F)$ vanishes on an affine $s$-dimension linear plane contained in the subspace

$$
\left\{\left(x_{1}, \ldots, x_{n}\right) \in \mathbb{F}_{q}^{n} \mid x_{i}=0 \text { for all } i>m\right\} .
$$

Then $\theta(F)$ must be a non-degenerate form in at least $6+s$ variables.

Proof. If $F$ is bottomless we conclude by Theorem 3 that $F$ has a nontrivial zero. Thus, $F$ must be $\Omega$-bottomed and consequently we may assume that $F$ is $\omega$-reduced for some $\omega>5$.

Suppose that $\theta(F)$ is non-degenerate with variables $x_{1}, \ldots, x_{m}$ explicit 
in $\theta(F)$ and $m \leq 5+s$. After a change of variables we may assume that $\theta(F)$ vanishes on the subspace

$$
\left\{\left(x_{1}, \ldots, x_{n}\right) \in \mathbb{F}_{q}^{n} \mid x_{i}=0 \text { for all } 1 \leq i \leq 5\right\} .
$$

If $\theta(F)$ vanishes on this space, but is not the zero polynomial, then it has a non-singular zero. Consequently, $\theta(F)$ has a non-trivial zero by Hensel's Lemma (see Lemma 6 below).

Otherwise, every monomial of $\theta(F)$ has at least one of $x_{1}, \ldots, x_{5}$ as a factor. Let $A \in \mathcal{O}_{\mathbb{K}}^{n \times n}$ be such that

$$
(A)_{i j}= \begin{cases}\pi & \text { for } 1 \leq i \leq 5 \text { and } i=j \\ 1 & \text { for } 6 \leq i \leq n \text { and } i=j \\ 0 & \text { for } i \neq j\end{cases}
$$

Then $\pi^{-1} F(A \mathbf{x})$ has coefficients in $\mathcal{O}_{\mathbb{K}}$ and we conclude

$$
F \succ \pi^{-1} F(A \mathbf{x}) \text {. }
$$

Note that for every degree larger than five there are non-zero forms which are vanishing everywhere, but do not possess a non-singular zero. An example is the form

$$
\left(x_{1} x_{2}^{3}+x_{1}^{3} x_{2}\right) x_{1} x_{2} \cdots x_{l} \in \mathbb{F}_{2}\left[x_{1}, \ldots, x_{l}\right] .
$$

The next lemma shows in particular that $s \geq 1$. For a system comprising forms $f_{1}, \ldots, f_{r}$ over $\mathbb{F}_{q}$ we shall denote the set of its projective zeros by $Z\left(f_{1}, \ldots, f_{r}\right)$.

Lemma 5 (Chevalley-Warning Theorem). Let $f_{1}, \ldots, f_{r}$ be forms of degree $d_{1}, \ldots, d_{r}$ over $\mathbb{F}_{q}$ in $n$ variables. If $n>d_{1}+\cdots+d_{r}$ we have

$$
\left|Z\left(f_{1}, \ldots, f_{r}\right)\right| \geq \frac{q^{n-d_{1}-\cdots-d_{r}}-1}{q-1} .
$$

A proof of this classical result can be found in [7]. Lemmas 4 and 5 yield the following consequence.

Corollary 1. Let $F$ be a quintic form in at least 26 variables over $\mathcal{O}_{\mathbb{K}}$ that does not have a non-trivial zero. Let $s$ be as defined in Lemma 4 . We then have

$$
|Z(\theta(F))| \geq \frac{q^{s+1}-1}{q-1} .
$$

A zero of $\theta(F)$ is not sufficient for a non-trivial zero of $F$, instead we require a non-singular zero. Once we have found one, we can apply the version of Hensel's Lemma given below. 
Lemma 6 (Hensel's Lemma). Let $F \in \mathcal{O}_{\mathbb{K}}\left[x_{1}, \ldots, x_{n}\right]$. If $\theta(F)$ has a non-singular zero, then $F$ has a non-trivial zero in $\mathbb{K}^{n}$.

For a discussion of Hensel's Lemma see [12], for example.

3.2. Proof of Theorem 1. Suppose that $F$ is a quintic form with $n \geq 26$ variables over a $p$-adic field $\mathbb{K}$ with residue class field of cardinality $q>9$. We denote the linear span of vectors $\mathbf{v}_{1}, \ldots, \mathbf{v}_{l} \in \mathbb{F}_{q}^{n}$ by $\left\langle\mathbf{v}_{1}, \ldots, \mathbf{v}_{l}\right\rangle$.

By Lemma 4 we may assume that $\theta(F)$ is a non-degenerate form in at least $6+s$ variables, where $s$ is the maximal affine dimension of a linear subspace of $Z(f)$. More precisely, $s$ is the maximal non-negative integer among those defined in Lemma 4. For ease of notation we shall write $f$ for the reduction $\theta(F)$.

Suppose that $f$ does not have a non-singular zero. We show that there are at least four linearly independent zeros

$$
\mathbf{z}_{1}, \mathbf{z}_{2}, \mathbf{z}_{3}, \mathbf{z}_{4} \in Z(f) \text { such that }\left\langle\mathbf{z}_{i}, \mathbf{z}_{j}\right\rangle \nsubseteq Z(f)
$$

for all $1 \leq i<j \leq 4$. Hence the form

$$
g\left(x_{1}, x_{2}, x_{3}, x_{4}\right):=f\left(x_{1} \mathbf{z}_{1}+x_{2} \mathbf{z}_{2}+x_{3} \mathbf{z}_{3}+x_{4} \mathbf{z}_{4}\right)
$$

must be of a certain shape. In particular, certain coefficients of $g$ do not vanish. We then prove the existence of a non-singular zero of $g$, contrary to our assumption. This is achieved by considering successively larger subspaces of $\left\langle\mathbf{z}_{1}, \mathbf{z}_{2}, \mathbf{z}_{3}, \mathbf{z}_{4}\right\rangle$ and sieving out forms possessing nonsingular zeros.

As a first step, we prove that there are five distinct non-zero vectors

$$
\mathbf{z}_{1}, \ldots, \mathbf{z}_{5} \in Z(f)
$$

such that $\mathbf{z}_{1}, \mathbf{z}_{2}, \mathbf{z}_{3}$ are linearly independent and $f$ does not vanish on any plane spanned by two vectors of one of the quadruples

$$
\left\{\mathbf{z}_{1}, \mathbf{z}_{2}, \mathbf{z}_{3}, \mathbf{z}_{i}\right\} \quad \text { where } i=4,5 .
$$

In order to establish this, we begin by showing that there are three distinct subspaces $V_{1}, V_{2}, V_{3} \subseteq Z(f)$ of maximal dimension and two zeros $\mathbf{z}_{1}, \mathbf{z}_{2} \in Z(f)$ such that

$$
\mathbf{z}_{1}, \mathbf{z}_{2} \notin \bigcup_{i=1}^{3} V_{i} \quad \text { and } \quad\left\langle\mathbf{z}_{1}, \mathbf{z}_{2}\right\rangle \nsubseteq Z(f) .
$$

Secondly, we prove the existence of a third zero $\mathbf{z}_{3} \in V_{3} \backslash\left(V_{1} \cup V_{2}\right)$ such that $\mathbf{z}_{1}, \mathbf{z}_{2}, \mathbf{z}_{3}$ are linearly independent. Thirdly, we show that there is a fourth zero $\mathbf{z}_{4} \in V_{2} \backslash V_{1}$ completing the first quadruple and finally, we will choose a fifth zero $\mathbf{z}_{5} \in V_{1}$ completing the second quadruple. 
For convenience, we first state a basic lemma and give the details of the argument outlined afterwards.

Lemma 7 ([18, Lemma 5.1]). Let $f$ be a quintic form over $\mathbb{F}_{q}$ possessing two distinct non-trivial zeros $\mathbf{z}_{1}$ and $\mathbf{z}_{2}$. Then $f$ either has a non-singular zero or

$$
f\left(x_{1} \mathbf{z}_{1}+x_{2} \mathbf{z}_{2}\right)=c_{12} x_{1}^{3} x_{2}^{2}+c_{21} x_{2}^{3} x_{1}^{2}
$$

and $c_{12} c_{21}=0$. If, in addition, $\left|\left\langle\mathbf{z}_{1}, \mathbf{z}_{2}\right\rangle \cap Z(f)\right| \geq 3$, then $f\left(x_{1} \mathbf{z}_{1}+x_{2} \mathbf{z}_{2}\right)$ either possesses a non-singular zero or is the zero polynomial.

Proof. We write

$f\left(x_{1} \mathbf{z}_{1}+x_{2} \mathbf{z}_{2}\right)=a_{1} x_{1}^{5}+b_{12} x_{1}^{4} x_{2}+c_{12} x_{1}^{3} x_{2}^{2}+c_{21} x_{2}^{3} x_{1}^{2}+b_{21} x_{2}^{4} x_{1}+a_{2} x_{2}^{5}$.

We may assume that $\mathbf{z}_{1}$ and $\mathbf{z}_{2}$ are singular zeros and hence

$$
f\left(x_{1} \mathbf{z}_{1}+x_{2} \mathbf{z}_{2}\right)=\left(c_{12} x_{1}+c_{21} x_{2}\right) x_{1}^{2} x_{2}^{2} .
$$

If $c_{12} c_{21} \neq 0$ then $\left(-c_{21}, c_{12}\right)$ is a non-singular zero and otherwise $\left\langle\mathbf{z}_{1}, \mathbf{z}_{2}\right\rangle \cap Z(f)=\left\{\mathbf{z}_{1}, \mathbf{z}_{2}\right\}$ or $\left\langle\mathbf{z}_{1}, \mathbf{z}_{2}\right\rangle \subseteq Z(f)$.

Since $f$ has at least 6 variables, Lemma 5 yields a non-trivial zero and thus we may assume $s \geq 1$. By Corollary 1 we have

$$
|Z(f)|>\frac{4\left(q^{s}-1\right)}{q-1},
$$

provided $q \geq 4$. Thus we can pick four distinct subspaces

$$
V_{1}, V_{2}, V_{3}, V_{4} \subseteq Z(f)
$$

such that $V_{i}$ is of maximal dimension for $1 \leq i \leq 4$. By equation (201) we can choose an additional zero $\mathbf{z}_{1} \in Z(f) \backslash \bigcup_{i=1}^{4} V_{i}$. We set $S_{3}:=\bigcup_{i=1}^{3} V_{i}$ and show that there exists a vector $\mathbf{z}_{2} \in V_{4} \backslash S_{3}$ such that $\left\langle\mathbf{z}_{1}, \mathbf{z}_{2}\right\rangle \nsubseteq Z(f)$. Suppose by the contrary that

$$
\text { for all } \mathbf{z} \in V_{4} \backslash S_{3} \text { we have }\left\langle\mathbf{z}_{1}, \mathbf{z}\right\rangle \subseteq Z(f) .
$$

If $V_{4} \cap S_{3}=\{0\}$, then (21) contradicts the maximality of $V_{4}$ and otherwise we shall argue as follows. Let $\mathbf{s} \in V_{4} \cap S_{3}$ be arbitrary. As $V_{4}$ is distinct from $S_{3}$ we can choose a non-zero vector $\mathbf{v} \in V_{4} \backslash S_{3}$ and consider the projective line $L_{\mathbf{s}}:=\langle\mathbf{v}, \mathbf{s}\rangle$. Since $\mathbf{v} \notin S_{3}$, the projective line $L_{\mathbf{s}}$ can not contain two vectors of $V_{i}$ for each $1 \leq i \leq 3$. Thus the intersection $L_{\mathbf{s}} \cap S_{3}$ contains at most three non-zero points. On the other hand, since $q \geq 5$, there are at least three points $\mathbf{p}_{1}, \mathbf{p}_{2}, \mathbf{p}_{3} \in L_{\mathbf{s}}$ not contained in $S_{3}$. It follows from our assumption (21) that $\left\langle\mathbf{z}_{1}, \mathbf{p}_{i}\right\rangle \subseteq Z(f)$ for all $1 \leq i \leq 3$. 
Lemma 8. Let $f$ be a quintic form over $\mathbb{F}_{q}$ without a non-singular zero, $L$ a projective line, $\mathbf{z}$ a non-zero point not on $L$ and $\mathbf{p}_{1}, \mathbf{p}_{2}, \mathbf{p}_{3} \in L$ three distinct non-zero points. Assume that

$$
\left\langle\mathbf{p}_{i}, \mathbf{z}\right\rangle \subseteq Z(f) \quad \text { for all } 1 \leq i \leq 3 .
$$

Then $\langle L, \mathbf{z}\rangle \subseteq Z(f)$.

Proof. Let $\mathbf{x} \in\langle L, \mathbf{z}\rangle$ and $\mathbf{x} \notin \bigcup_{i=1}^{3}\left\langle\mathbf{p}_{i}, \mathbf{z}\right\rangle$. There exists a projective line $H$ in $\langle L, \mathbf{z}\rangle$ through $\mathbf{x}$ that does not contain $\mathbf{z}$. Since we have assumed that $\mathbf{x} \notin\left\langle\mathbf{p}_{i}, \mathbf{z}\right\rangle$ and $\left\langle\mathbf{p}_{i}, \mathbf{z}\right\rangle$ has co-dimension 1 in $\langle L, \mathbf{z}\rangle$, the line $H$ intersects $\left\langle\mathbf{p}_{i}, \mathbf{z}\right\rangle$ in exactly one point $\mathbf{s}_{i}$, say, for each $1 \leq i \leq 3$. Since $\bigcap_{i=1}^{3}\left\langle\mathbf{p}_{i}, \mathbf{z}\right\rangle=\mathbf{z}$ and $\mathbf{z} \notin H$, we conclude that there are at least three distinct points, namely $\mathbf{s}_{i}$ for $1 \leq i \leq 3$, in $H$ that are contained in $Z(f)$. By Lemma 7 we have $H \subseteq Z(f)$ and hence $\mathbf{x} \in Z(f)$. We conclude that $\langle L, \mathbf{z}\rangle \subseteq Z(f)$.

By applying Lemma 8 we have $\left\langle\mathbf{z}_{1}, V_{4}\right\rangle \subseteq Z(f)$, contrary to the maximality of the dimension of $V_{4}$. We conclude that there are three non-identical subspaces $V_{1}, V_{2}, V_{3} \subseteq Z(f)$ of maximal dimension and two zeros $\mathbf{z}_{1}, \mathbf{z}_{2} \notin \bigcup_{i=1}^{3} V_{i}$ such that

$$
\left\langle\mathbf{z}_{1}, \mathbf{z}_{2}\right\rangle \cap Z(f)=\left\{\mathbf{z}_{1}, \mathbf{z}_{2}\right\} .
$$

As mentioned above we shall proceed by proving the existence of a third vector $\mathbf{z}_{3} \in V_{3} \backslash\left(V_{1} \cup V_{2}\right)$ with the property $\left\langle\mathbf{z}_{i}, \mathbf{z}_{j}\right\rangle \nsubseteq Z(f)$ for all $1 \leq i<j \leq 3$. Suppose by the contrary that for every $\mathbf{z} \in V_{3} \backslash\left(V_{1} \cup V_{2}\right)$ at least one of the following holds

$$
\left\langle\mathbf{z}, \mathbf{z}_{1}\right\rangle \subseteq Z(f) \quad \text { or } \quad\left\langle\mathbf{z}, \mathbf{z}_{2}\right\rangle \subseteq Z(f) .
$$

We set $S_{2}:=V_{1} \cup V_{2}$ for shorter notation and shall argue that we may assume $S_{2} \cap V_{3}=\{0\}$. Suppose there exists at least one non-zero vector $\mathbf{s} \in S_{2} \cap V_{3}$. We then pick a vector $\mathbf{v} \in V_{3} \backslash S_{2}$ and define for any vector $\mathbf{s} \in S_{2} \cap V_{3}$ the projective line $L_{\mathbf{s}}:=\langle\mathbf{s}, \mathbf{v}\rangle$. We show that

$$
\left\langle L_{\mathbf{s}}, \mathbf{z}_{1}\right\rangle \subseteq Z(f) \text { or }\left\langle L_{\mathbf{s}}, \mathbf{z}_{2}\right\rangle \subseteq Z(f) .
$$

Since $\mathbf{v} \notin S_{2}$, neither two vectors of the subspace $V_{1}$ nor two of the subspace $V_{2}$ can be contained in $L_{\mathbf{s}}$. Thus there are at least 5 projective points in $L_{\mathbf{s}} \backslash S_{2}$, provided $q \geq 6$. By our assumption (22) there are three points $\mathbf{p}_{1}, \mathbf{p}_{2}, \mathbf{p}_{3}$ among them such that $\left\langle\mathbf{p}_{i}, \mathbf{z}_{k}\right\rangle \subseteq Z(f)$ for all $1 \leq i \leq 3$ and a certain $1 \leq k \leq 2$. Equation (23) then follows from Lemma 8 and thus, we have that for every $\mathbf{z} \in V_{3}$ at least one of the following holds

$$
\left\langle\mathbf{z}, \mathbf{z}_{1}\right\rangle \subseteq Z(f) \quad \text { or } \quad\left\langle\mathbf{z}, \mathbf{z}_{2}\right\rangle \subseteq Z(f)
$$


Lemma 9. Let $f$ be a quintic form over $\mathbb{F}_{q}$ without a non-singular zero, $V \subseteq Z(f)$ an $m$-dimensional subspace where $m \geq 2$ and $\mathbf{z}_{1}, \ldots, \mathbf{z}_{k}$ non-trivial zeros not contained in $V$. We assume $q \geq 2 k$ and that there exists for any projective plane $W \subseteq V$ of co-dimension 1 an index $i \in\{1, \ldots, k\}$ such that $\left\langle W, \mathbf{z}_{i}\right\rangle \subseteq Z(f)$. Then there exists an index $i \in\{1, \ldots, k\}$ such that

$$
\left\langle V, \mathbf{z}_{i}\right\rangle \subseteq Z(f)
$$

Proof. We write $\left[x_{1}: \cdots: x_{m}\right]$ for a projective point in $V$. Since $m \geq 2$ we can define the following subspaces

$$
W_{(a, b)}:=\left\{\left[x_{1}: \cdots: a x_{m-1}: b x_{m-1}\right] \mid x_{i} \in \mathbb{F}_{q} \text { for } 1 \leq i \leq m\right\}
$$

for $(a, b) \in\left(\{1\} \times \mathbb{F}_{q}\right) \cup\{(0,1)\}$.

Since $q \geq 2 k$ there are at least $2 k+1$ subspaces $W_{(a, b)}$. Thus we may assume that there are at least three subspaces, $W_{1}, W_{2}, W_{3}$ say, among these and a zero $\mathbf{z} \in\left\{\mathbf{z}_{1}, \ldots, \mathbf{z}_{k}\right\}$ such that

$$
\left\langle W_{i}, \mathbf{z}\right\rangle \subseteq Z(f) \quad \text { for } 1 \leq i \leq 3 .
$$

We shall complete the proof of this lemma by following Leep and Yeomans [[18], Lemma 5.3]. For $W_{1}, W_{2}, W_{3}$ as above, we have

$$
\begin{aligned}
& \left\langle W_{i}, \mathbf{z}\right\rangle \cap\left\langle W_{j}, \mathbf{z}\right\rangle=\left\langle W_{i} \cap W_{j}, \mathbf{z}\right\rangle, \\
& \left\langle W_{i}, \mathbf{z}\right\rangle \cap\left\langle W_{j}, \mathbf{z}\right\rangle=\bigcap_{i=1}^{3}\left\langle W_{i}, \mathbf{z}\right\rangle
\end{aligned}
$$

for any $1 \leq i<j \leq 3$. We notice that for equation (25) we have for each pair $i \neq j$ with $\left\langle W_{i}, \mathbf{z}\right\rangle$ and $\left\langle W_{j}, \mathbf{z}\right\rangle$ two non-identical $m$-dimensional planes and that $\left\langle W_{i} \cap W_{j}, \mathbf{z}\right\rangle$ is an $m-1$ dimensional plane. Equation (26) follows from (25) and the fact that

$$
W_{i} \cap W_{j}=\bigcap_{i=1}^{3} W_{i} \text { for distinct } i, j .
$$

Let $\mathbf{x}$ be a point in $\langle V, \mathbf{z}\rangle \backslash \bigcup_{i=1}^{3}\left\langle W_{i}, \mathbf{z}\right\rangle$. We observe that $\bigcap_{i=1}^{3} W_{i}$ has codimension 2 in $V$. Thus, we conclude by (25) and (26) that $\bigcap_{i=1}^{3}\left\langle W_{i}, \mathbf{z}\right\rangle$ has co-dimension 2 in $\langle V, \mathbf{z}\rangle$. Hence we can choose a projective line $H$ through the point $\mathbf{x}$ that does not intersect with $\bigcap_{i=1}^{3}\left\langle W_{i}, \mathbf{z}\right\rangle$. Since $\mathbf{x} \notin\left\langle W_{i}, \mathbf{z}\right\rangle$ and $\left\langle W_{i}, \mathbf{z}\right\rangle$ has co-dimension 1 in $\langle V, \mathbf{z}\rangle$, we conclude that there exists for each $i$ a point $\mathbf{p}_{i} \in\left\langle W_{i}, \mathbf{z}\right\rangle \cap H$. Since $\left\langle W_{i}, \mathbf{z}\right\rangle \subseteq$ $Z(f)$ and $H$ does not intersect $\bigcap_{i=1}^{3}\left\langle W_{i}, \mathbf{z}\right\rangle$ there are at least three distinct non-trivial zeros of $f$ on $H$. Thus we conclude by Lemma 7 that $\langle V, \mathbf{z}\rangle \subseteq Z(f)$. 
We apply Lemma 9 to (24) and thus, we have

$$
\left\langle V_{3}, \mathbf{z}_{1}\right\rangle \subseteq Z(f) \quad \text { or } \quad\left\langle V_{3}, \mathbf{z}_{2}\right\rangle \subseteq Z(f) .
$$

However, this contradicts the maximality of the dimension of $V_{3}$. Moreover, the vectors $\mathbf{z}_{1}, \mathbf{z}_{2}, \mathbf{z}_{3}$ are linearly independent, since by Lemma 7 there are at most two zeros on the projective line $\left\langle\mathbf{z}_{1}, \mathbf{z}_{2}\right\rangle$. Thus we have found three linearly independent vectors $\mathbf{z}_{1}, \mathbf{z}_{2}, \mathbf{z}_{3}$ such that

$$
\left\langle\mathbf{z}_{i}, \mathbf{z}_{j}\right\rangle \nsubseteq Z(f) \text { for all } 1 \leq i<j \leq 3 .
$$

We show that there exists a fourth vector $\mathbf{z}_{4} \in V_{2} \backslash V_{1}$ such that

$$
\left\langle\mathbf{z}_{i}, \mathbf{z}_{j}\right\rangle \nsubseteq Z(f) \quad \text { for all } 1 \leq i<j \leq 4 .
$$

Suppose by the contrary that for all $\mathbf{z} \in V_{2} \backslash V_{1}$ at least one of the following holds

$$
\left\langle\mathbf{z}, \mathbf{z}_{1}\right\rangle \subseteq Z(f), \quad\left\langle\mathbf{z}, \mathbf{z}_{2}\right\rangle \subseteq Z(f) \quad \text { or } \quad\left\langle\mathbf{z}, \mathbf{z}_{3}\right\rangle \subseteq Z(f) .
$$

We shall argue that there is no loss of generality if we assume $V_{1} \cap V_{2}=$ $\{0\}$. As there exists a point $\mathbf{v} \in V_{2} \backslash V_{1}$ we consider for any vector $\mathbf{s} \in V_{2} \cap V_{1}$ the plane $L_{\mathbf{s}}:=\langle\mathbf{s}, \mathbf{v}\rangle$. We show that

$$
\left\langle L_{\mathbf{s}}, \mathbf{z}_{1}\right\rangle \subseteq Z(f), \quad\left\langle L_{\mathbf{s}}, \mathbf{z}_{2}\right\rangle \subseteq Z(f) \quad \text { or } \quad\left\langle L_{\mathbf{s}}, \mathbf{z}_{3}\right\rangle \subseteq Z(f) .
$$

Since $q \geq 7$ there are at least 7 projective points in $L_{\mathbf{s}}$ not contained in $V_{1}$. Thus, by (27) there are three points $\mathbf{p}_{1}, \mathbf{p}_{2}, \mathbf{p}_{3}$ among them such that $\left\langle\mathbf{p}_{i}, \mathbf{z}_{k}\right\rangle \subseteq Z(f)$ for all $1 \leq i \leq 3$ and a certain $1 \leq k \leq 3$. By Lemma 8 , we have that for every $\mathbf{z} \in V_{2}$ at least one of the following holds

$$
\left\langle\mathbf{z}, \mathbf{z}_{1}\right\rangle \subseteq Z(f), \quad\left\langle\mathbf{z}, \mathbf{z}_{2}\right\rangle \subseteq Z(f) \quad \text { or } \quad\left\langle\mathbf{z}, \mathbf{z}_{3}\right\rangle \subseteq Z(f) .
$$

It then follows in conjunction with Lemma 9 that

$$
\left\langle V_{2}, \mathbf{z}_{1}\right\rangle \subseteq Z(f), \quad\left\langle V_{2}, \mathbf{z}_{2}\right\rangle \subseteq Z(f) \quad \text { or } \quad\left\langle V_{2}, \mathbf{z}_{3}\right\rangle \subseteq Z(f) .
$$

However, any of those contradicts the maximality of the dimension of $V_{2}$ and hence we may assume the existence of a vector $\mathbf{z}_{4} \in V_{2} \backslash V_{1}$ such that

$$
\left\langle\mathbf{z}_{i}, \mathbf{z}_{j}\right\rangle \nsubseteq Z(f) \text { for all } 1 \leq i<j \leq 4 .
$$

We show that there exists a fifth vector $\mathbf{z}_{5} \in V_{1}$ such that

$$
\left\langle\mathbf{z}_{i}, \mathbf{z}_{5}\right\rangle \nsubseteq Z(f) \text { for all } 1 \leq i \leq 3 .
$$

Suppose by the contrary that for all $\mathbf{z} \in V_{1}$ at least one of the conditions in equation (27) holds. By Lemma 9 this implies

$$
\left\langle V_{1}, \mathbf{z}_{1}\right\rangle \subseteq Z(f), \quad\left\langle V_{1}, \mathbf{z}_{2}\right\rangle \subseteq Z(f) \quad \text { or } \quad\left\langle V_{1}, \mathbf{z}_{3}\right\rangle \subseteq Z(f) .
$$


However, any of these contradicts the maximality of the dimension of $V_{1}$ and thus we conclude that there is a vector $\mathbf{z}_{5} \in V_{1}$ such that

$$
\left\langle\mathbf{z}_{i}, \mathbf{z}_{5}\right\rangle \nsubseteq Z(f) \text { for all } 1 \leq i \leq 3 .
$$

In summary, we have shown that there are two quadruples of zeros,

$$
\mathbf{z}_{1}, \mathbf{z}_{2}, \mathbf{z}_{3}, \mathbf{z}_{4} \text { and } \mathbf{z}_{1}, \mathbf{z}_{2}, \mathbf{z}_{3}, \mathbf{z}_{5}
$$

such that $f$ does not vanish on any two-dimensional plane spanned by two zeros of one quadruple. Moreover, we know that $\mathbf{z}_{1}, \mathbf{z}_{2}, \mathbf{z}_{3}$ are linearly independent. We will now estimate the number of zeros of $f$ in $\left\langle\mathbf{z}_{1}, \mathbf{z}_{2}, \mathbf{z}_{3}\right\rangle$.

Lemma 10. Let $f$ be a quintic form over $\mathbb{F}_{q}$ with three linearly independent zeros $\mathbf{z}_{1}, \mathbf{z}_{2}, \mathbf{z}_{3} \in Z(f)$ such that $\left\langle\mathbf{z}_{i}, \mathbf{z}_{j}\right\rangle \nsubseteq Z(f)$ for all $1 \leq i<j \leq 3$. Then the following holds.

If $q \geq 17$, then $f$ has a non-singular zero. If $11 \leq q<17$, it possesses a non-singular zero or $\left|\left\langle\mathbf{z}_{1}, \mathbf{z}_{2}, \mathbf{z}_{3}\right\rangle \cap Z(f)\right|=3$ holds. If $q<11$ it has a non-singular zero or $\left|\left\langle\mathbf{z}_{1}, \mathbf{z}_{2}, \mathbf{z}_{3}\right\rangle \cap Z(f)\right| \leq 4$ holds.

The last inequality is sharp. For instance, $2 x_{1}^{3} x_{2}^{2}+2 x_{1}^{3} x_{3}^{2}+4 x_{2}^{3} x_{3}^{2}+x_{1} x_{2} x_{3}\left(5 x_{1}^{2}+6 x_{2}^{2}+2 x_{3}^{2}+x_{1} x_{2}+x_{1} x_{3}+x_{2} x_{3}\right)$ is a form over $\mathbb{F}_{7}$ possessing exactly four singular zeros, namely

$$
\langle(1,0,0)\rangle,\langle(0,1,0)\rangle,\langle(0,0,1)\rangle,\langle(1,6,2)\rangle .
$$

Proof. Suppose that $f$ does not have a non-singular zero. Thus we can write $f\left(x_{1} \mathbf{z}_{1}+x_{2} \mathbf{z}_{2}+x_{3} \mathbf{z}_{3}\right)$ as

$$
x_{1} x_{2} x_{3} Q\left(x_{1}, x_{2}, x_{3}\right)+\sum_{1 \leq i<j \leq 3} c_{i j} x_{i}^{3} x_{j}^{2}+c_{j i} x_{j}^{3} x_{i}^{2}
$$

where $Q\left(x_{1}, x_{2}, x_{3}\right)$ is a quadratic form. By applying Lemma 7 to any two variables of $f\left(x_{1} \mathbf{z}_{1}+x_{2} \mathbf{z}_{2}+x_{3} \mathbf{z}_{3}\right)$ we have $c_{i j} c_{j i}=0$ for all $1 \leq i<$ $j \leq 3$. Since $f$ does not vanish on any of the projective lines $\left\langle\mathbf{z}_{i}, \mathbf{z}_{j}\right\rangle$ with $1 \leq i<j \leq 3$, we have either

$$
c_{i j} \neq 0 \quad \text { or } \quad c_{j i} \neq 0 \quad \text { for all } 1 \leq i<j \leq 3 .
$$

Hence, we see after permuting the variables that $f\left(x_{1} \mathbf{z}_{1}+x_{2} \mathbf{z}_{2}+x_{3} \mathbf{z}_{3}\right)$ takes one of the following shapes

$$
\begin{aligned}
& t_{1}\left(x_{1}, x_{2}, x_{3}\right)=c_{12} x_{1}^{3} x_{2}^{2}+c_{13} x_{1}^{3} x_{3}^{2}+c_{23} x_{2}^{3} x_{3}^{2}+x_{1} x_{2} x_{3} Q\left(x_{1}, x_{2}, x_{3}\right), \\
& t_{2}\left(x_{1}, x_{2}, x_{3}\right)=c_{12} x_{1}^{3} x_{2}^{2}+c_{31} x_{3}^{3} x_{1}^{2}+c_{23} x_{2}^{3} x_{3}^{2}+x_{1} x_{2} x_{3} Q\left(x_{1}, x_{2}, x_{3}\right),
\end{aligned}
$$

where $Q\left(x_{1}, x_{2}, x_{3}\right)$ is a quadratic form and $c_{12}, c_{13}, c_{23}$ and $c_{31}$ are all non-zero coefficients.

It has been proved by Leep and Yeomans [18] using the Lang-Weil 
Bound that $f\left(x_{1} \mathbf{z}_{1}+x_{2} \mathbf{z}_{2}+x_{3} \mathbf{z}_{3}\right)$ has always a non-singular zero, provided $q \geq 43$. Heath-Brown [14] has extended this to prime values of $q \geq 17$.

Similarly, we show by computer calculations that $f$ has a non-singular zero for $q=25,27,32$. In each case there are, after an appropriate rescaling of both, the forms $t_{1}, t_{2}$ and the variables, just 6 degrees of freedom. A computer program can verify the existence of a nonsingular zero for each form $t_{1}$, respectively each form $t_{2}$, by successively testing points in $\mathbb{F}_{q}^{3}$.

If $q<17$ it can be checked by an analogous computer calculation that $t_{1}$ and $t_{2}$ either possess a non-singular zero or that the bound on $\left|\left\langle\mathbf{z}_{1}, \mathbf{z}_{2}, \mathbf{z}_{3}\right\rangle \cap Z(f)\right|$ holds.

Lemma 10 establishes Theorem 1, provided $q \geq 17$. Moreover, it shows that not both quadruples $\mathbf{z}_{1}, \mathbf{z}_{2}, \mathbf{z}_{3}, \mathbf{z}_{4}$ and $\mathbf{z}_{1}, \mathbf{z}_{2}, \mathbf{z}_{3}, \mathbf{z}_{5}$ can consist of linearly dependent vectors. Thus we may assume, after renaming, that we have linearly independent vectors $\mathbf{z}_{1}, \mathbf{z}_{2}, \mathbf{z}_{3}, \mathbf{z}_{4}$ such that

$$
\left\langle\mathbf{z}_{i}, \mathbf{z}_{j}\right\rangle \nsubseteq Z(f) \text { for all } 1 \leq i<j \leq 4
$$

We write $f\left(x_{1} \mathbf{z}_{1}+x_{2} \mathbf{z}_{2}+x_{3} \mathbf{z}_{3}+x_{4} \mathbf{z}_{4}\right)$ as

$$
\sum_{i \neq j} a_{i j} x_{i}^{3} x_{j}^{2}+\sum_{\substack{k \neq i, j \\ i<j}} b_{i j k} x_{i} x_{j} x_{k}^{3}+\sum_{\substack{i \neq j, k \\ j<k}} c_{i j k} x_{i} x_{j}^{2} x_{k}^{2}+\sum_{\substack{l \neq i, j, k \\ i<j<k}} d_{i j k l} x_{i} x_{j} x_{k} x_{l}^{2},
$$

where $1 \leq i, j, k \leq 4$. By applying Lemma 7 and since $f$ does not vanish on any of the projective lines $\left\langle\mathbf{z}_{i}, \mathbf{z}_{j}\right\rangle$, we conclude that for each pair $(i, j)$ with $i \neq j$ exactly one of $a_{i j}$ and $a_{j i}$ is zero. It then follows that, after a permutation of the variables, the form (29) can take only four different shapes. If we write $h$ for

$$
\begin{aligned}
& a_{23} x_{2}^{3} x_{3}^{2}+a_{24} x_{2}^{3} x_{4}^{2}+a_{34} x_{3}^{3} x_{4}^{2}+ \\
& \sum_{\substack{k \neq i, j \\
i<j}} b_{i j k} x_{i} x_{j} x_{k}^{3}+\sum_{\substack{i \neq j, k \\
j<k}} c_{i j k} x_{i} x_{j}^{2} x_{k}^{2}+\sum_{\substack{l \neq i, j, k \\
i<j<k}} d_{i j k l} x_{i} x_{j} x_{k} x_{l}^{2}
\end{aligned}
$$

those are

$$
\begin{aligned}
& g_{1}:=a_{12} x_{1}^{3} x_{2}^{2}+a_{13} x_{1}^{3} x_{3}^{2}+a_{14} x_{1}^{3} x_{4}^{2}+h, \\
& g_{2}:=a_{12} x_{1}^{3} x_{2}^{2}+a_{31} x_{3}^{3} x_{1}^{2}+a_{14} x_{1}^{3} x_{4}^{2}+h, \\
& g_{3}:=a_{12} x_{1}^{3} x_{2}^{2}+a_{13} x_{1}^{3} x_{3}^{2}+a_{41} x_{4}^{3} x_{1}^{2}+h, \\
& g_{4}:=a_{21} x_{2}^{3} x_{1}^{2}+a_{13} x_{1}^{3} x_{3}^{2}+a_{41} x_{4}^{3} x_{1}^{2}+h .
\end{aligned}
$$


As indicated it has been checked on a computer that each of those forms has a non-singular zero, provided $9<q \leq 16$. We briefly describe the assembling process.

Along the way, we have already excluded, via Lemma 7, all forms that have a non-singular zero on one of the projective lines $\left\langle\mathbf{z}_{i}, \mathbf{z}_{j}\right\rangle$ for some $1 \leq i<j \leq 4$. Furthermore, we know from the proof of Lemma 10 all forms which do not have a non-singular zero in one of the subspaces

$$
\left\langle\mathbf{z}_{i}, \mathbf{z}_{j}, \mathbf{z}_{k}\right\rangle \text { for some } 1 \leq i<j<k \leq 4 \text {. }
$$

Note that $g_{1}, g_{2}, g_{3}$ and $g_{4}$ restricted to such a subspace are, after permuting the variables, equal to $t_{1}$ or $t_{2}$ as stated in the proof of Lemma 10. The computer programs for $g_{1}, g_{2}, g_{3}$ and $g_{4}$ are analogous. Suppose $g_{s}$ for some $1 \leq s \leq 4$ is one of these cases. We save the rearranged coefficients of those forms of shape $t_{1}$, respectively $t_{2}$, without a nonsingular zero in four multidimensional arrays

$$
A_{i j k}[\star, \star] \quad \text { where } 1 \leq i<j<k \leq 4
$$

such that they represent the coefficients of $g_{s}$ restricted to the subspace $\left\langle\mathbf{z}_{i}, \mathbf{z}_{j}, \mathbf{z}_{k}\right\rangle$. Thus, every set of coefficients of the form $\left.g_{s}\right|_{\left\langle\mathbf{z}_{i}, \mathbf{z}_{j}, \mathbf{z}_{k}\right\rangle}$ without a non-singular zero corresponds to a line $A_{i j k}[r, \star]$.

We use these data to construct all remaining forms by combining data in these arrays and four additional degrees of freedom. Let $r_{i j k}$ denote the $r_{i j k}$-th line of $A_{i j k}[\star, \star]$ for $1 \leq i<j<k \leq 4$. The non-negative integers $r_{123}, r_{124}, r_{134}, r_{234}$, provided the corresponding lines are compatible with respect to the coefficients they share, determine a form

$$
C\left(r_{123}, r_{124}, r_{134}, r_{234}\right)
$$

in four variables, $x_{1}, x_{2}, x_{3}, x_{4}$ say, with each monomial in at most three variables. Thus any relevant form of shape $g_{s}$ can be written as

$$
\begin{aligned}
& C\left(r_{123}, r_{124}, r_{134}, r_{234} ; a, b, c, d\right) \\
& \quad=C\left(r_{123}, r_{124}, r_{134}, r_{234}\right)+x_{1} x_{2} x_{3} x_{4}\left(a x_{1}+b x_{2}+c x_{3}+d x_{4}\right) .
\end{aligned}
$$

For all admissible $r_{123}, r_{124}, r_{134}, r_{234}$ and for all $a, b, c, d \in \mathbb{F}_{q}$ we then search for a non-singular zero $\left(x_{1}, x_{2}, x_{3}, x_{4}\right) \in \mathbb{F}_{q}^{4}$ of

$$
C\left(r_{123}, r_{124}, r_{134}, r_{234} ; a, b, c, d\right)
$$

by trying points successively. To do this efficiently, one can rescale both the forms and variables. For instance, rescale $g_{1}, g_{2}, g_{3}$ such that

$$
a_{12}=1, \quad a_{23}=1, \quad a_{34}=1
$$

and $g_{4}$ such that

$$
a_{21}=1, \quad a_{23}=1, \quad a_{34}=1 .
$$


It is easier to choose a rescaling that is compatible with the one used in Lemma 10 (and hence with the data in the arrays $\left.A_{i j k}[\star, \star]\right)$. Besides these considerations, we put a general effort on implementing the algorithm efficiently.

The source code of the C++ program may be found in the appendix. The data and the source code used in the assembling process are available at [10]. This completes the proof of Theorem 1 .

Note that apart from the computer checks we have not used any assumption other than $q>5$. For $q=8,9$ it is likely that one can also find by a computer search a non-singular zero of every form of the shapes $g_{1}, g_{2}, g_{3}$ and $g_{4}$. Whereas the case $q=7$ seems more doubtful than $q=8,9$, one can easily find counterexamples, for instance of shape $g_{1}$, for $q=5$ using the same algorithm. 


\section{Systems comprising a Cubic and a Quadratic Form}

We give a condensed account of Zahid's work [26] and incorporate a slicing result by Lecerf to prove Theorem 2 ,

Recall that $\mathbb{K}$ denotes a $p$-adic field with residue class field $\mathbb{F}_{q}$ and ring of integers $\mathcal{O}_{\mathbb{K}}$. As previously mentioned, we write $\pi$ for a uniformiser of $\mathcal{O}_{\mathbb{K}}$. The $h$-invariant of a system comprising forms $F_{1}, \ldots, F_{r} \in$ $\mathcal{O}_{\mathbb{K}}[\mathbf{x}]$, denoted by $h\left(F_{1}, \ldots, F_{r}\right)$, is the smallest non-negative integer $h$ such that we can write

$$
F_{i}(\mathbf{x})=L_{1} H_{1, i}(\mathbf{x})+\cdots+L_{h} H_{h, i}(\mathbf{x}) \quad(\bmod \pi)
$$

where $L_{1}, \ldots, L_{h}$ are linear and $H_{1, i}, \ldots, H_{h, i}$ suitable forms over $\mathbb{F}_{q}$ and this holds for all $1 \leq i \leq r$.

Suppose that $q>37$ and $\mathbb{F}_{q}$ has characteristic at least 13 . Let $C\left(x_{1}, \ldots, x_{n}\right)$ be a cubic and $Q\left(x_{1}, \ldots, x_{n}\right)$ a quadratic form over $\mathbb{K}$ with $n \geq 14$ variables. As we are interested in a zero, we may assume from now on that $C$ and $Q$ are defined over $\mathcal{O}_{\mathbb{K}}$.

By Schmidt's minimisation procedure (see Theorem 3) we may assume that the system comprising $C$ and $Q$ is $\left(\omega_{1}, \omega_{2}\right)$-reduced for some $\omega_{1}>3$ and $\omega_{2}>2$. Zahid then shows that

$$
h(Q)>2, \quad h(C-L Q)>3 \quad \text { and } \quad h(C-L Q, Q)>5
$$

for all linear forms $L(\mathbf{x}) \in \mathcal{O}_{\mathbb{K}}[\mathbf{x}]$. These inequalities can be proved similarly to Lemma 4 .

We denote by $f$ and $g$ the reduction of $C$ and $Q$, respectively, over the residue class field. Using the Chevalley-Warning Theorem (Lemma 5) and the Lang-Weil Bound (Lemma 12 below) he then shows that there exists a non-zero vector $\mathbf{e} \in \mathbb{F}_{q}^{n}$ such that

$$
f(\mathbf{e})=g(\mathbf{e})=0 \quad \text { and } \quad \nabla g(\mathbf{e}) \neq 0 .
$$

Thus we may consider instead the equivalent system

$$
\begin{aligned}
& f(\mathbf{x})=x_{1} f_{2}\left(\mathbf{x}_{3}\right)+f_{3}\left(\mathbf{x}_{2}\right) \\
& g(\mathbf{x})=x_{1} x_{2}+g_{2}\left(\mathbf{x}_{3}\right)
\end{aligned}
$$

where $\mathbf{x}_{l}=\left(x_{l}, x_{l+1}, \ldots, x_{n}\right)$.

Following Zahid we show that there exists $\mathbf{x}_{2} \in \mathbb{F}_{q}^{n-1}$ such that $x_{2} \neq 0$ and $\left(-x_{2}^{-1} g_{2}\left(\mathbf{x}_{3}\right), \mathbf{x}_{2}\right)$ is a common non-singular zero of $f$ and $g$. Recall that a common non-singular zero of $f$ and $g$ is a zero $\mathbf{x}$ such that $\nabla f(\mathbf{x})$ and $\nabla g(\mathbf{x})$ are linearly independent. By applying the following variant 
of Hensel's Lemma we then find a common non-trivial zero of $C$ and $Q$.

Lemma 11 (Hensel's Lemma for systems of forms). Let $F_{1}, \ldots, F_{r}$ be a system of forms over $\mathcal{O}_{\mathbb{K}}$. Suppose that $\theta\left(F_{1}\right), \ldots, \theta\left(F_{r}\right)$ possess a common non-singular zero. Then $F_{1}, \ldots, F_{r}$ have a common non-trivial zero.

Suppose first that $f_{2}=0$. It is enough to show that there exists a non-singular zero $\mathbf{x}_{2} \in \mathbb{F}_{q}^{n-1}$ of $f_{3}$ such that $x_{2} \neq 0$. If $\operatorname{deg}_{x_{2}} f_{3}\left(\mathbf{x}_{2}\right)<3$, this is relatively straightforward, provided $q>5$. If $\operatorname{deg}_{x_{2}} f_{3}\left(\mathbf{x}_{2}\right)=3$, it is straightforward to find a non-singular zero $\mathbf{a} \in \mathbb{F}_{q}^{n-1}$ of $f_{3}$. If $a_{2}=0$, then Zahid sets $\mathbf{e}_{3}:=\mathbf{a}$ and chooses an additional vector $\mathbf{e}_{4} \in \mathbb{F}_{q}^{n-1}$ such that

$f_{3}\left(X \mathbf{e}_{2}+Y \mathbf{e}_{3}+Z \mathbf{e}_{4}\right):=c X^{3}+Y^{2} u(X, Z)+Y v(X, Z)+w(X, Z)+d Z^{3}$

where $c, d \neq 0, u(X, 1) \neq 0$ and $\operatorname{deg}_{X} w \leq 2$, provided $q>5$. If this form is absolutely irreducible he uses the following variant of the Lang-Weil Bound to obtain a non-singular zero of $f_{3}$ such that $x_{2} \neq 0$, provided $q>3$.

Lemma 12 (Lemma 5.3, [26]). Let $N$ be the number of non-singular zeros of an absolutely irreducible polynomial of degree $d$ with two variables, defined over $\mathbb{F}_{q}$. Then $N$ satisfies

$$
N \geq q+1-\frac{1}{2}(d-1)(d-2)\lfloor 2 \sqrt{q}\rfloor .
$$

If $f_{3}\left(X \mathbf{e}_{2}+Y \mathbf{e}_{3}+Z \mathbf{e}_{4}\right)$ is not absolutely irreducible, it must factor as a product of a linear form over $\mathbb{F}_{q}$ and a quadratic form. It then follows from a short argument that we can find $\mathbf{x}_{2}$ as claimed.

Consequently, we may assume that $f_{2} \neq 0$. Zahid then defines

$$
H\left(\mathbf{x}_{2}\right)=x_{2} f_{3}\left(\mathbf{x}_{2}\right)-\left(f_{2} g_{2}\right)\left(\mathbf{x}_{3}\right)
$$

and by exploiting (30) establishes that $H$ is absolutely irreducible. Moreover, he shows that $\left(-x_{2}^{-1} g_{2}\left(\mathbf{x}_{3}\right), \mathbf{x}_{2}\right)$ is a common non-singular zero of $f$ and $g$, provided that $\mathbf{x}_{2}$ is a non-singular zero of $H$ such that $x_{2} \neq 0$. For $\xi \in \mathbb{F}_{q}^{3 n-5}$ we define the sliced polynomial

$\left.H\right|_{\xi}(X, Y):=H\left(\xi_{1}+X, \xi_{2}+\xi_{n} X+\xi_{2 n-4} Y, \ldots, \xi_{n-1}+\xi_{2 n-3} X+\xi_{3 n-5} Y\right)$.

We use the following variant of a result of Lecerf (see Corollary 8, [17]) to show that there exists a slice such that $\left.H\right|_{\xi}$ is absolutely irreducible. 
Lemma 13. Let $F \in \mathbb{F}_{q}\left[x_{2}, \ldots, x_{n}\right]$ be an absolutely irreducible polynomial of degree $d$. Assume that $\mathbb{F}_{q}$ has characteristic at least $d(d-1)+1$ and $q>3 d(d-1)+1$. Then there exists a slice $\xi \in \mathbb{F}_{q}^{3 n-5}$ such that the sliced polynomial $\left.F\right|_{\xi}$ is absolutely irreducible.

By Lemma 13 there exists a slice $\xi \in \mathbb{F}_{q}^{3 n-5}$ such that $\left.H\right|_{\xi}$ is absolutely irreducible, provided $\mathbb{F}_{q}$ has characteristic at least 13 and $q>37$. The $x_{2}$-component of $\left.H\right|_{\xi}$ can not be identically zero, as this would contradict the fact that $\left.H\right|_{\xi}$ is absolutely irreducible. Finally, we apply the Lang-Weil Bound as stated in Lemma 12 to show that there exists a non-singular zero of $H$ such that $x_{2} \neq 0$. This completes the proof.

Given there exists a suitable slice, the Lang-Weil Bound, as stated in Lemma 12, yields a non-singular zero as long as $q \geq 37$. If one could make sure that the sliced polynomial has one or more singular zeros, then its genus would decrease and the condition on $q$ could be weakened. 


\section{Appendix}

The following $\mathrm{C}++$ program serves as an example for the computer programs used in Chapter 3. For improved readability much of the data in the arrays A123, A124 and A134 has been removed. The data as well as the program source code can be accessed at [10].

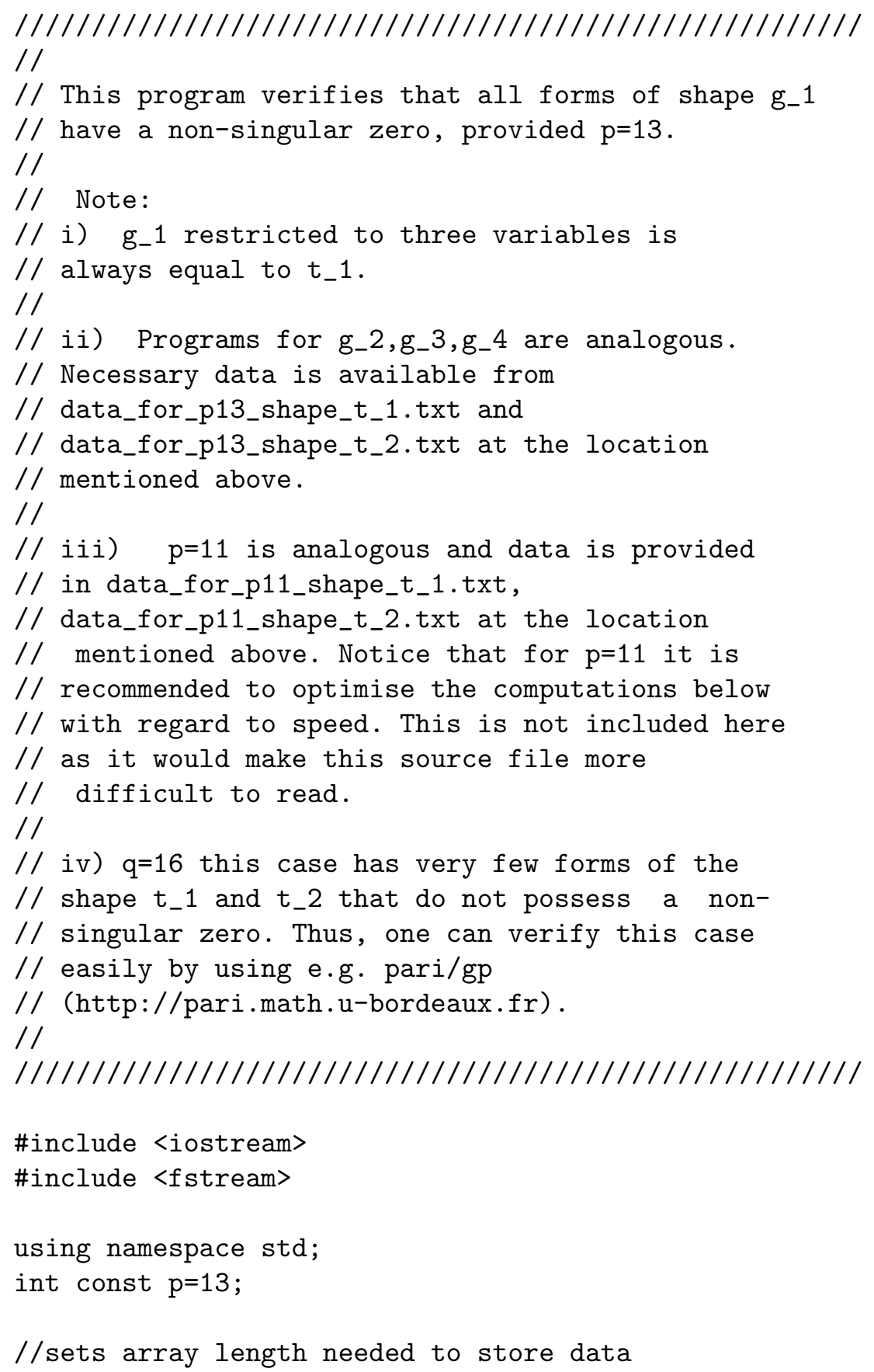


int const $\mathrm{r} 123=48$;

int const $r 124=576$;

int const $r 134=576$;

$/ / \log -f i l e$

ofstream cfile;

// Returns for coefficients (A[i][0],A[i][1],A[i][2], //A[i] [3], A [i] [4], A [i] [5], A [i] [6], A[i] [7], A[i] [8], $/ / A[j][1], A[j][2], A[j][3], A[j][4], A[j][5], A[j][6]$, $/ / A[j][7], A[j][8], A[k][1], A[k][3], A[k][4], A[k][5]$, $/ / A[k][6], A[k][7], A[k][8], A[1][3], A[1][4], A[1][5]$, $\left./ / A[1][6], A[1][7], A[1][8], d_{-} 1, d_{-} 2, d_{-} 3, d_{-} 4\right)$ the //value 1 if $\left(x_{-} 1, x_{-} 2, x_{-} 3, x_{-} 4\right)$ is a non-singular zero $/ /$ and 0 otherwise.

int nonsingular(int A123_i_0, int A123_i_1, int A123_i_2, int A123_i_3,int A123_i_4,int A123_i_5, int A123_i_6,

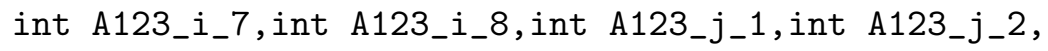
int $A 1233_{-} j_{3}$, int $A 1233_{-} j_{-} 4$, int $A 123$ _j_5, int $A 123_{-} j_{-} 6$, int $A 123_{-} j_{-} 7$, int $A 123_{-} j_{-} 8$, int $A 1344_{-} k_{-}$, int $A 1344_{-} k_{-} 3$, int $\mathrm{A} 1344_{-} \mathrm{k}_{-} 4$, int $\mathrm{A} 1344_{-} \mathrm{k}_{-} 5$, int $\mathrm{A} 134_{-} \mathrm{k}_{-} 6$, int $\mathrm{A} 1344_{-} \mathrm{k}_{-} 7$,

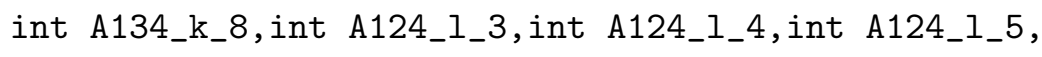
int $A 124 \_1 \_6$, int $A 124 \_{ }_{-} 7$, int $A 124 \_1 \_8$, int $d_{-} 1$, int $d_{-} 2$, int $\mathrm{d}_{-} 3$, int $\mathrm{d}_{-} 4$, int $\mathrm{x}_{-} 1$, int $\mathrm{x}_{-} 2$, int $\mathrm{x}_{-} 3$, int $\mathrm{x}_{-} 4$ ) \{

//first partial derivative int $\mathrm{A}=3 * \mathrm{~A} 123 \_\mathrm{i}_{-} 0 * \mathrm{x}_{-} 1 * \mathrm{x}_{-} 1 * \mathrm{x}_{-} 2 * \mathrm{x}_{-} 2+3 * \mathrm{~A} 1233_{-} \mathrm{i}_{-} 1 * \mathrm{x}_{-} 1 * \mathrm{x}_{-} 1 * \mathrm{x}_{-} 3 * \mathrm{x}_{-} 3$ $+\mathrm{x}_{-} 2 * \mathrm{x}_{-} 3 *\left(\mathrm{x}_{-} 1 * \mathrm{x}_{-} 1 * \mathrm{~A} 123 \_\mathrm{i}{ }_{-} 3+\mathrm{x}_{-} 1 * \mathrm{x}_{-} 2 * \mathrm{~A} 123 \_\mathrm{i}{ }_{-} 6+\right.$ $\mathrm{x} \_1 * \mathrm{x} \_3 * \mathrm{~A} 123 \_\mathrm{i}_{-} 7+\mathrm{x} \_2 * \mathrm{x} \_2 * \mathrm{~A} 123 \_\mathrm{i}_{-} 4+\mathrm{x} \_2 * \mathrm{x} \_3 * \mathrm{~A} 123 \_\mathrm{i}_{-} 8+$ $\left.\mathrm{x}_{-} 3 * \mathrm{x} \_3 * \mathrm{~A} 123 \_i_{-} 5\right)+\mathrm{x}_{-} 1 * \mathrm{x}_{-} 2 * \mathrm{x}_{-} 3 *\left(2 * \mathrm{x}_{-} 1 * \mathrm{~A} 1233_{-} i_{-} 3+\mathrm{x}_{-} 2 * \mathrm{~A} 123 \_\mathrm{i}_{-} 6\right.$ $\left.+\mathrm{x}_{-} 3 * \mathrm{~A} 123 \mathrm{i}_{-} 7\right)+3 * \mathrm{~A} 134 \mathrm{x}_{-} 1 * \mathrm{x}_{-} 1 * \mathrm{x}_{-} 1 * \mathrm{x}_{-} 4 * \mathrm{x}_{-} 4+$ $\mathrm{x}_{-} 3 * \mathrm{x}_{-} 4 *\left(\mathrm{x}_{-} 1 * \mathrm{x}_{-} 1 * \mathrm{~A} 1344_{-} \mathrm{k}_{-} 3+\mathrm{x}_{-} 1 * \mathrm{x}_{-} 3 * \mathrm{~A} 1344_{-} \mathrm{k}_{-} 6+\mathrm{x}_{-} 1 * \mathrm{x}_{-} 4 * \mathrm{~A} 134 \mathrm{x}_{-} \mathrm{k}\right.$ $\left.+\mathrm{x}_{-} 3 * \mathrm{x}_{-} 3 * \mathrm{~A} 134 \mathrm{x}_{-} \mathrm{k}_{-} 4+\mathrm{x}_{-} 3 * \mathrm{x}_{-} 4 * \mathrm{~A} 1344_{-} \mathrm{k}_{-} 8+\mathrm{x}_{-} 4 * \mathrm{x}_{-} 4 * \mathrm{~A} 1344_{-} \mathrm{k}_{-} 5\right)+$ $\mathrm{x}_{-} 1 * \mathrm{x}_{-} 3 * \mathrm{x}_{-} 4 *\left(2 * \mathrm{x}_{-} 1 * \mathrm{~A} 1344_{-} \mathrm{k}_{-} 3+\mathrm{x}_{-} 3 * \mathrm{~A} 134{ }_{-} \mathrm{k}_{-} 6+\mathrm{x}_{-} 4 * \mathrm{~A} 1344_{-} \mathrm{k}_{-} 7\right)+$

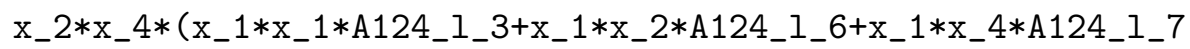
$\left.\left.+\mathrm{x}_{-} 2 * \mathrm{x}_{-} 2 * \mathrm{~A} 124{ }_{-} 1_{-} 4+\mathrm{x}_{-} 2 * \mathrm{x}_{-} 4 * \mathrm{~A} 1244_{-} 1_{-} 8+\mathrm{x}_{-} 4 * \mathrm{x}_{-} 4 * \mathrm{~A} 1244_{-}\right]_{-} 5\right)+$ $\left.\mathrm{x}_{-} 1 * \mathrm{x}_{-} 2 * \mathrm{x}_{-} 4 *\left(2 * \mathrm{x}_{-} 1 * \mathrm{~A} 124{ }_{-} 1_{-} 3+\mathrm{x}_{-} 2 * \mathrm{~A} 124{ }_{-} 1_{-} 6+\mathrm{x}_{-} 4 * \mathrm{~A} 1244_{-}\right]_{-} 7\right)+$ $\mathrm{d}_{-} 1 * \mathrm{x}_{-} 1 * \mathrm{x}_{-} 2 * \mathrm{x}_{-} 3 * \mathrm{x}_{-} 4+$ $\left(\mathrm{d}_{-} 1 * \mathrm{x}_{-} 1+\mathrm{d}_{-} 2 * \mathrm{x}_{-} 2+\mathrm{d}_{-} 3 * \mathrm{x}_{-} 3+\mathrm{d}_{-} 4 * \mathrm{x}_{-} 4\right) * \mathrm{x}_{-} 2 * \mathrm{x}_{-} 3 * \mathrm{x}_{-} 4$;

//second partial derivative int $\mathrm{B}=2 * \mathrm{~A} 123 \_i_{-} 0 * \mathrm{x}_{-} 1 * \mathrm{x}_{-} 1 * \mathrm{x}_{-} 1 * \mathrm{x}_{-} 2+3 * \mathrm{~A} 123 \_\mathrm{i}_{-} 2 * \mathrm{x}_{-} 2 * \mathrm{x}_{-} 2 * \mathrm{x}_{-} 3 * \mathrm{x} \_3$ $+\mathrm{x}_{-} 1 * \mathrm{x}_{-} 3 *\left(\mathrm{x}_{-} 1 * \mathrm{x}_{-} 1 * \mathrm{~A} 123 \_i_{-} 3+\mathrm{x}_{-} 1 * \mathrm{x}_{-} 2 * \mathrm{~A} 123 \_i_{-} 6+\right.$ 
$\mathrm{x} \_1 * \mathrm{x} \_3 * \mathrm{~A} 123 \_\mathrm{i} \_7+\mathrm{x} \_2 * \mathrm{x} \_2 * \mathrm{~A} 123$ i_ $4+\mathrm{x} \_2 * \mathrm{x} \_3 * \mathrm{~A} 123$ i_8+

$\mathrm{x} \_3 * \mathrm{x} \_3 * \mathrm{~A} 123$ i $\left.{ }_{-} 5\right)+\mathrm{x} \_1 * \mathrm{x} \_2 * \mathrm{x} \_3 *\left(\mathrm{x} \_1 * \mathrm{~A} 123\right.$ _i_6+2 $2 * \mathrm{x}_{-} 2 * \mathrm{~A} 123$ _i_4+ $\mathrm{x} \_3 * \mathrm{~A} 123$ _i_8) $+3 * \mathrm{~A} 123$ _j_1 $1 * \mathrm{x} \_2 * \mathrm{x} \_2 * \mathrm{x} \_4 * \mathrm{x} \_4+$

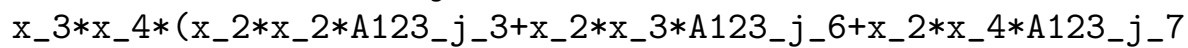

$\left.+\mathrm{x}_{-} 3 * \mathrm{x}_{-} 3 * \mathrm{~A} 1233_{-} \mathrm{j}_{-} 4+\mathrm{x} \_3 * \mathrm{x}_{-} 4 * \mathrm{~A} 123_{-} \mathrm{j}_{-} 8+\mathrm{x}_{-} 4 * \mathrm{x}_{-} 4 * \mathrm{~A} 1233_{-} \mathrm{j}_{-} 5\right)+$

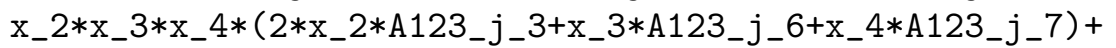

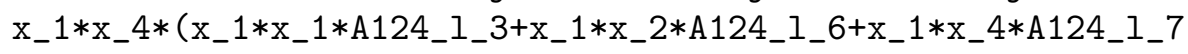

$\left.+\mathrm{x} \_2 * \mathrm{x} \_2 * \mathrm{~A} 124 \_1 \_4+\mathrm{x} \_2 * \mathrm{x} \_4 * \mathrm{~A} 124{ }_{-} 1_{-} 8+\mathrm{x}_{-} 4 * \mathrm{x}_{-} 4 * \mathrm{~A} 124{ }_{-}{ }_{-} 5\right)+$

$\mathrm{x} \_1 * \mathrm{x} \_2 * \mathrm{x} \_4 *\left(\mathrm{x} \_1 * \mathrm{~A} 124{ }_{-} 1_{-} 6+2 * \mathrm{x} \_2 * \mathrm{~A} 124{ }_{-} 1_{-} 4+\mathrm{x} \_4 * \mathrm{~A} 124 \_1 \_8\right)+$

$\mathrm{d} \_2 * \mathrm{x} \_1 * \mathrm{x} \_2 * \mathrm{x} \_3 * \mathrm{x} \_4+$

$\left(\mathrm{d} \_1 * \mathrm{x} \_1+\mathrm{d} \_2 * \mathrm{x} \_2+\mathrm{d} \_3 * \mathrm{x} \_3+\mathrm{d} \_4 * \mathrm{x} \_4\right) * \mathrm{x} \_1 * \mathrm{x} \_3 * \mathrm{x} \_4$;

//third partial derivative

int $\mathrm{C}=2 * \mathrm{~A} 123 \_\mathrm{i}_{-} 1 * \mathrm{x}_{-} 1 * \mathrm{x}_{-} 1 * \mathrm{x}_{-} 1 * \mathrm{x} \_3+2 * \mathrm{~A} 123 \_\mathrm{i}_{-} 2 * \mathrm{x} \_2 * \mathrm{x} \_2 * \mathrm{x} \_2 * \mathrm{x} \_3$

$+\mathrm{x}_{-} 1 * \mathrm{x} \_2 *\left(\mathrm{x}_{-} 1 * \mathrm{x}_{-} 1 * \mathrm{~A} 123 \_\mathrm{i}_{-} 3+\mathrm{x} \_1 * \mathrm{x} \_2 * \mathrm{~A} 123\right.$ i ${ }_{-} 6+$

$\mathrm{x} \_1 * \mathrm{x} \_3 * \mathrm{~A} 123 \_\mathrm{i} \_7+\mathrm{x} \_2 * \mathrm{x} \_2 * \mathrm{~A} 123$ i_ $4+\mathrm{x} \_2 * \mathrm{x} \_3 * \mathrm{~A} 123$ _i_8+

$\left.\mathrm{x} \_3 * \mathrm{x} \_3 * \mathrm{~A} 123 \_\mathrm{i} \_5\right)+\mathrm{x} \_1 * \mathrm{x} \_2 * \mathrm{x} \_3 *\left(\mathrm{x} \_1 * \mathrm{~A} 123 \_\mathrm{i}_{-} 7+\mathrm{x} \_2 * \mathrm{~A} 123\right.$ i_8+

$\left.2 * \mathrm{x}_{-} 3 * \mathrm{~A} 1233_{-} \mathrm{i}_{-} 5\right)+3 * \mathrm{~A} 1233_{-} \mathrm{j}_{-} 2 * \mathrm{x}_{-} 3 * \mathrm{x}_{-} 3 * \mathrm{x}_{-} 4 * \mathrm{x}_{-} 4+$

$\mathrm{x}_{-} 2 * \mathrm{x}_{-} 4 *\left(\mathrm{x}_{-} 2 * \mathrm{x}_{-} 2 * \mathrm{~A} 1233_{-} \mathrm{j}_{-} 3+\mathrm{x}_{-} 2 * \mathrm{x}_{-} 3 * \mathrm{~A} 1233_{-} \mathrm{j}_{-} 6+\right.$

$\mathrm{x}_{-} 2 * \mathrm{x}_{-} 4 * \mathrm{~A} 1233_{-} \mathrm{j}_{-} 7+\mathrm{x} \_3 * \mathrm{x}_{-} 3 * \mathrm{~A} 1233_{-} \mathrm{j}_{-} 4+\mathrm{x} \_3 * \mathrm{x}_{-} 4 * \mathrm{~A} 1233_{-} \mathrm{j}_{-} 8+$

$\left.\mathrm{x}_{-} 4 * \mathrm{x}_{-} 4 * \mathrm{~A} 1233_{-} \mathrm{j}_{-} 5\right)+\mathrm{x}_{-} 2 * \mathrm{x}_{-} 3 * \mathrm{x}_{-} 4 *\left(\mathrm{x} \_2 * \mathrm{~A} 1233_{-} \mathrm{j}_{-} 6+2 * \mathrm{x} \_3 * \mathrm{~A} 1233_{-} \mathrm{j}_{-} 4+\right.$

$\left.\mathrm{x}_{-} 4 * \mathrm{~A} 123-\mathrm{j} \_8\right)+\mathrm{x}_{-} 1 * \mathrm{x} \_4 *\left(\mathrm{x}_{-} 1 * \mathrm{x}_{-} 1 * \mathrm{~A} 1344_{-} \mathrm{k}_{-} 3+\mathrm{x}_{-} 1 * \mathrm{x} \_3 * \mathrm{~A} 1344_{-} \mathrm{k} \_6+\right.$

$\mathrm{x} \_1 * \mathrm{x} \_4 * \mathrm{~A} 134 \mathrm{k}_{-} 7+\mathrm{x} \_3 * \mathrm{x} \_3 * \mathrm{~A} 134 \mathrm{x}_{-} 4+\mathrm{x} \_3 * \mathrm{x} \_4 * \mathrm{~A} 134 \mathrm{k}_{-} 8+$

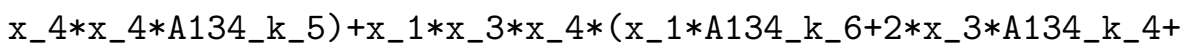

$\mathrm{x}_{-} 4 * \mathrm{~A} 134$, $\left.\mathrm{z}_{-} 8\right)+\mathrm{d}_{-} 3 * \mathrm{x}_{-} 1 * \mathrm{x}_{-} 2 * \mathrm{x}_{-} 3 * \mathrm{x}_{-} 4+$

$\left(\mathrm{d} \_1 * \mathrm{x} \_1+\mathrm{d} \_2 * \mathrm{x} \_2+\mathrm{d} \_3 * \mathrm{x} \_3+\mathrm{d}_{-} 4 * \mathrm{x}_{-} 4\right) * \mathrm{x}_{-} 1 * \mathrm{x}_{-} 2 * \mathrm{x} \_4$;

//fourth partial derivative

int $\mathrm{D}=2 * \mathrm{~A} 1233_{-} \mathrm{j}_{-} 1 * \mathrm{x}_{-} 2 * \mathrm{x}_{-} 2 * \mathrm{x}_{-} 2 * \mathrm{x}_{-} 4+2 * \mathrm{~A} 123{ }_{-} \mathrm{j}_{-} 2 * \mathrm{x}_{-} 3 * \mathrm{x}_{-} 3 * \mathrm{x}_{-} 3 * \mathrm{x}_{-} 4$

$+\mathrm{x}_{-} 2 * \mathrm{x}_{-} 3 *\left(\mathrm{x}_{-} 2 * \mathrm{x}_{-} 2 * \mathrm{~A} 123 \_\mathrm{j} \_3+\mathrm{x} \_2 * \mathrm{x} \_3 * \mathrm{~A} 123\right.$-j_6+

$\mathrm{x}_{-} 2 * \mathrm{x}_{-} 4 * \mathrm{~A} 1233_{-} \mathrm{j}_{-} 7+\mathrm{x} \_3 * \mathrm{x}_{-} 3 * \mathrm{~A} 1233_{-} \mathrm{j}_{-} 4+\mathrm{x} \_3 * \mathrm{x}_{-} 4 * \mathrm{~A} 123 \_\mathrm{j}_{-} 8+$

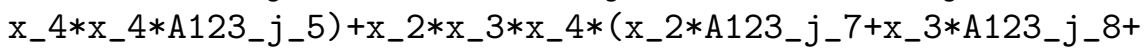

$\left.2 * \mathrm{x}_{-} 4 * \mathrm{~A} 123{ }_{-} \mathrm{j}_{-} 5\right)+2 * \mathrm{~A} 1344_{-} \mathrm{k}_{-} 1 * \mathrm{x} \_1 * \mathrm{x} \_1 * \mathrm{x} \_1 * \mathrm{x} \_4+$

$\mathrm{x} \_1 * \mathrm{x} \_3 *\left(\mathrm{x}_{-} 1 * \mathrm{x} \_1 * \mathrm{~A} 134{ }_{-} \mathrm{k}_{-} 3+\mathrm{x} \_1 * \mathrm{x} \_3 * \mathrm{~A} 134{ }_{-} \mathrm{k} \_6+\right.$

$\mathrm{x} \_1 * \mathrm{x} \_4 * \mathrm{~A} 134 \_\mathrm{k} \_7+\mathrm{x} \_3 * \mathrm{x} \_3 * \mathrm{~A} 134{ }_{-} \mathrm{k}_{-} 4+\mathrm{x} \_3 * \mathrm{x} \_4 * \mathrm{~A} 134$ _

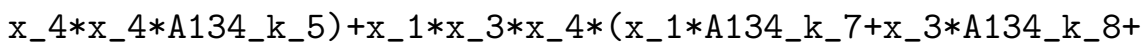

$\left.2 * \mathrm{x}_{-} 4 * \mathrm{~A} 134 \mathrm{x}_{-} 5\right)+\mathrm{x} \_1 * \mathrm{x} \_2 *\left(\mathrm{x}_{-} 1 * \mathrm{x} \_1 * \mathrm{~A} 124{ }_{-}{ }_{-} 3+\mathrm{x}_{-} 1 * \mathrm{x} \_2 * \mathrm{~A} 124\right.$ _1_6+

$\mathrm{x} \_1 * \mathrm{x} \_4 * \mathrm{~A} 124 \_1 \_7+\mathrm{x} \_2 * \mathrm{x} \_2 * \mathrm{~A} 124{ }_{-}{ }_{-}\left(4+\mathrm{x} \_2 * \mathrm{x} \_4 * \mathrm{~A} 124\right.$ _1_8+

$\left.\mathrm{x}_{-} 4 * \mathrm{x} \_4 * \mathrm{~A} 124 \_1 \_5\right)+\mathrm{x} \_1 * \mathrm{x} \_2 * \mathrm{x}_{-} 4 *\left(\mathrm{x} \_1 * \mathrm{~A} 124{ }_{-}{ }_{-} 7+\mathrm{x} \_2 * \mathrm{~A} 124\right.$ _1_8+

$\left.2 * \mathrm{x}_{-} 4 * \mathrm{~A} 124 \_1 \_5\right)+\mathrm{d}_{-} 4 * \mathrm{x}_{-} 1 * \mathrm{x}_{-} 2 * \mathrm{x}_{-} 3 * \mathrm{x} \_4$

$+\left(\mathrm{d}_{-} 1 * \mathrm{x} \_1+\mathrm{d} \_2 * \mathrm{x} \_2+\mathrm{d} \_3 * \mathrm{x} \_3+\mathrm{d}_{-} 4 * \mathrm{x} \_4\right) * \mathrm{x} \_1 * \mathrm{x} \_2 * \mathrm{x} \_3$;

if $((A) \% p !=0)\{$ return $1 ;\}$;

if $((\mathrm{B}) \% \mathrm{p} !=0)\{$ return $1 ;\}$;

if $((\mathrm{C}) \% \mathrm{p} !=0)\{$ return $1 ;\}$; 
if $((D) \% p !=0)$ rreturn $1 ;\}$;

return 0 ;

\}

int main () \{

// file would have saved the message that counterexamples //have been found cfile.open ("shape_g_1.txt");

//We rescale those coefficient in front //of $x_{-} 1^{\wedge} 3 x_{-} 2^{\wedge} 2, x_{-} 2^{\wedge} 3 x_{-} 3^{\wedge} 2, x_{-} 3^{\wedge} 3 x_{-} 4^{\wedge} 2$ in $g_{-} 1$.

//Lines in array represent $\mathrm{g}_{-} 1 \mathrm{I}_{-}\left\{\mathrm{y}_{-} 1, \mathrm{y}_{-} 2, \mathrm{y}_{-} 3\right\}$

//without a non-singular zero.

//We use the compatible rescaling

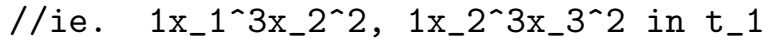

int $\mathrm{A} 123[\mathrm{r} 123][9]=\{$

$\{1,1,1,2,7,11,2,2,5\}$,

...

\}

//We use the compatible rescaling ie. $1 x_{-} 1 \leadsto 3 x_{-} 2^{\wedge} 2$ in $t_{-} 1$ int $\mathrm{A} 124[\mathrm{r} 124][9]=\{$

$\{1,1,1,2,7,11,2,2,5\}$,

$\cdots$

\};

//We use the compatible rescaling ie. $1 x_{-} 2^{\wedge} 3 x_{-} 3^{\wedge} 2$ in $t_{-} 1$

int $\mathrm{A} 134[\mathrm{r} 134][9]=\{$

$\{1,1,1,2,7,11,2,2,5\}$,

...

\};

$/ /$ Note that $\mathrm{A} 234=\mathrm{A} 123$.

//indicates whether a non-singular zero has been found int found $=0$;

//Loops used in the assembling process: 


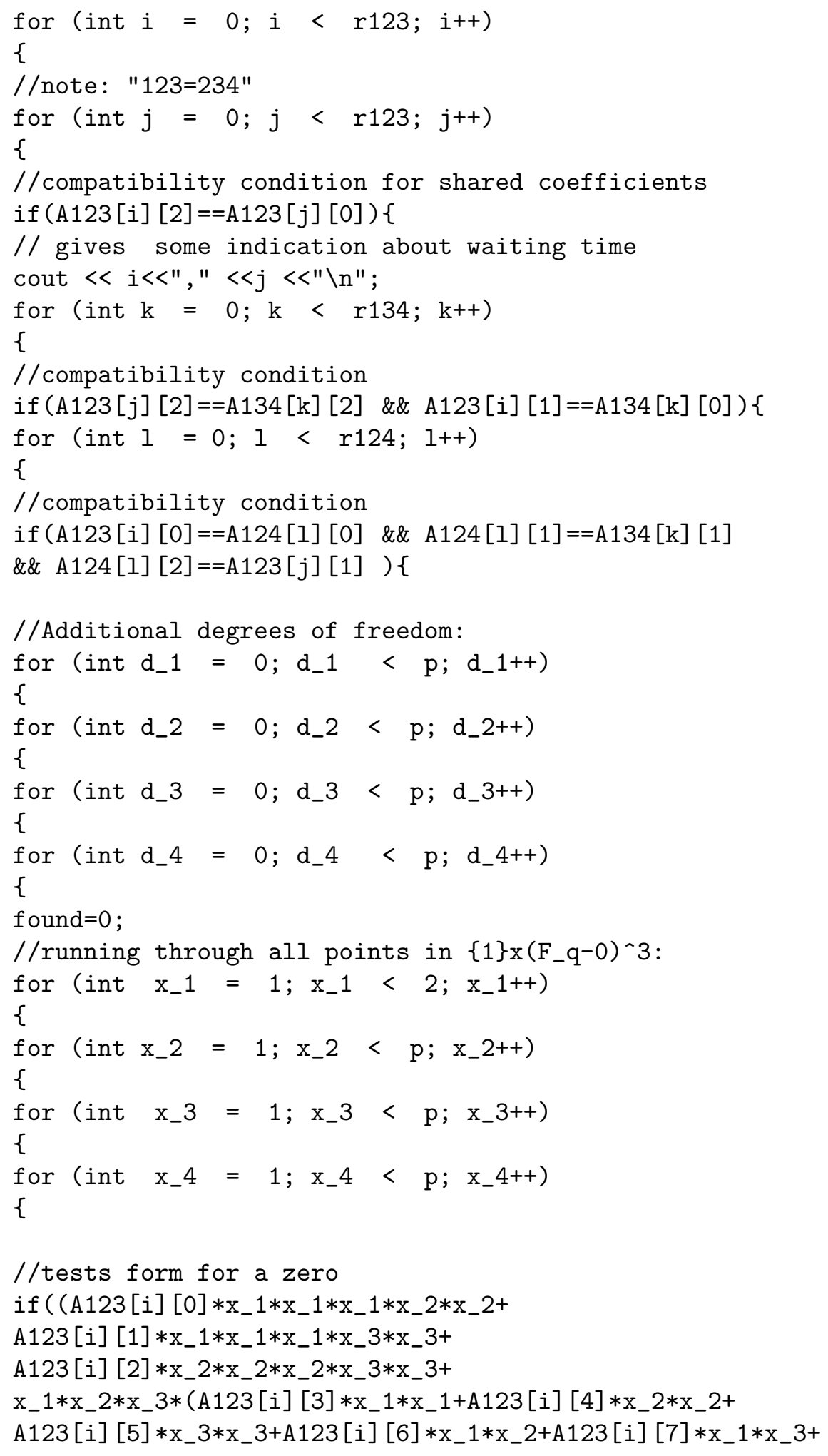




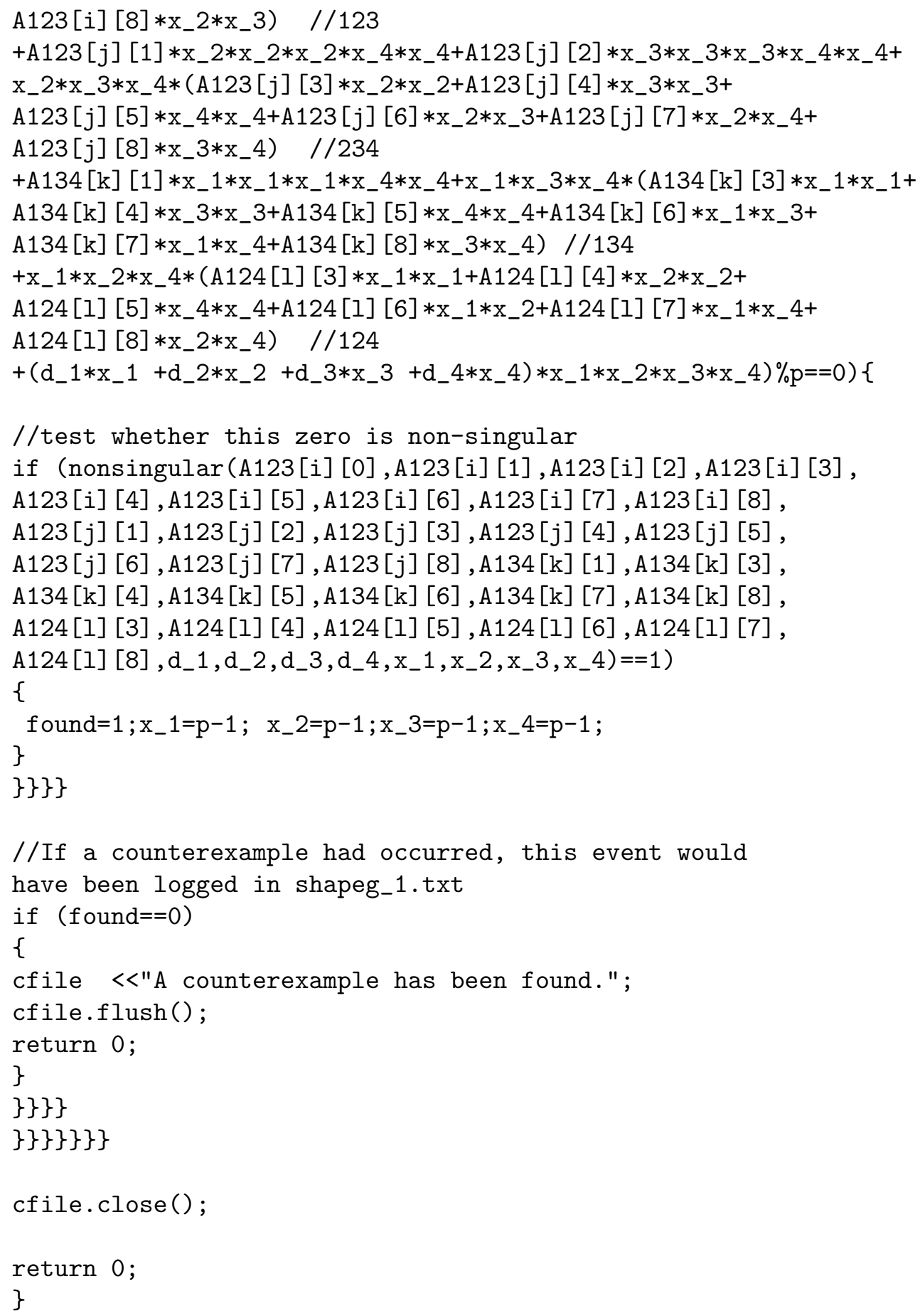




\section{REFERENCES}

1. J. Ax and S. Kochen, Diophantine problems over local fields. I, Amer. J. Math. 87 (1965), 605-630.

2. B. J. Birch and D. J. Lewis, p-adic forms, J. Indian Math. Soc. (N.S.) 23 (1959), 11-32 (1960).

3. Systems of three quadratic forms, Acta Arith. 10 (1964/1965), 423442.

4. B. J. Birch, D. J. Lewis, and T. G. Murphy, Simultaneous quadratic forms, Amer. J. Math. 84 (1962), 110-115.

5. R. Brauer, A note on systems of homogeneous algebraic equations, Bull. Amer. Math. Soc. 51 (1945), 749-755.

6. S. S. Brown, Bounds on transfer principles for algebraically closed and complete discretely valued fields, Mem. Amer. Math. Soc. 15 (1978), no. 204, iv+92.

7. C. Chevalley and E. Warning, Bemerkung zur vorstehenden arbeit, Abhandlungen aus dem Mathematischen Seminar der Universitaet Hamburg 11 (1935), $76-83$ (German).

8. H. Davenport and D. J. Lewis, Homogeneous additive equations, Proc. Roy. Soc. Ser. A 274 (1963), 443-460.

9. V. B. Demyanov, Pairs of quadratic forms over a complete field with discrete norm with a finite field of residue classes, Izv. Akad. Nauk SSSR. Ser. Mat. 20 (1956), 307-324.

10. J. H. Dumke, p-adic zeros of quintic forms, submitted, arXiv:1308.0999v2 (2013).

11. Quartic forms in many variables, submitted, arXiv: 1405.7064 (2014).

12. M. J. Greenberg, Lectures on forms in many variables, W. A. Benjamin, Inc., New York-Amsterdam, 1969.

13. H. Hasse, Über die Darstellbarkeit von Zahlen durch quadratische Formen im Körper der rationalen Zahlen., Journal reine angew.Math. 152 (1923), 129-148.

14. D. R. Heath-Brown, Zeros of p-adic forms, Proc. Lond. Math. Soc. (3) 100 (2010), no. 2, 560-584.

15. Z_ Zeros of systems of p-adic quadratic forms, Compos. Math. 146 (2010), no. 2, 271-287.

16. R. R. Laxton and D. J. Lewis, Forms of degrees 7 and 11 over p-adic fields, Proc. Sympos. Pure Math., Vol. VIII, Amer. Math. Soc., Providence, R.I., 1965, pp. 16-21.

17. G. Lecerf, Improved dense multivariate polynomial factorization algorithms, J. Symbolic Comput. 42 (2007), no. 4, 477-494.

18. D. B. Leep and C. C. Yeomans, Quintic forms over p-adic fields, J. Number Theory 57 (1996), no. 2, 231-241.

19. D. J. Lewis, Cubic homogeneous polynomials over p-adic number fields, Ann. of Math. (2) 56 (1952), 473-478.

20. W. M. Schmidt, Simultaneous p-adic zeros of quadratic forms, Monatsh. Math. 90 (1980), no. 1, 45-65.

21. S. E. Schuur, On systems of three quadratic forms, Acta Arith. 36 (1980), no. 4, $315-322$.

22. G. Terjanian, Un contre-exemple à une conjecture d'Artin, C. R. Acad. Sci. Paris Sér. A-B 262 (1966), A612. 
23. T. D. Wooley, On the local solubility of Diophantine systems, Compositio Math. 111 (1998), no. 2, 149-165.

24. (2008), no. 1, 25-35.

25. J. Zahid, Zeros of p-adic forms, J. Number Theory 129 (2009), no. 10, 24392456.

26. Simultaneous zeros of a cubic and quadratic form, J. Lond. Math. Soc. (2) 84 (2011), no. 3, 612-630. 